\title{
Numerical computation of rotation numbers of quasi-periodic planar curves
}

\author{
Alejandro Luque Jordi Villanueva \\ March 24, 2009* \\ Departament de Matemàtica Aplicada I, \\ Universitat Politècnica de Catalunya, \\ Diagonal 647, 08028 Barcelona (Spain). \\ alejandro.luque@upc.edu jordi.villanueva@upc.edu
}

\begin{abstract}
Recently, a new numerical method has been proposed to compute rotation numbers of analytic circle diffeomorphisms, as well as derivatives with respect to parameters, that takes advantage of the existence of an analytic conjugation to a rigid rotation. This method can be directly applied to the study of invariant curves of planar twist maps by simply projecting the iterates of the curve onto a circle. In this work we extend the methodology to deal with general planar maps. Our approach consists in computing suitable averages of the iterates of the map that allow to obtain a new curve for which the direct projection onto a circle is well posed. Furthermore, since our construction does not use the invariance of the quasiperiodic curve under the map, it can be applied to more general contexts. We illustrate the method with several examples.
\end{abstract}

PACS: 02.30.-f; 02.60.-x; 02.70.-c

Keywords: Invariant curves; Rotation number; Non-twist maps; Numerical Approximation.

*This paper was published in: Physica D 238 (2009) pp. 2025-2044. 


\section{Contents}

1 Introduction $\quad 2$

2 Exposition of methods $\quad 5$

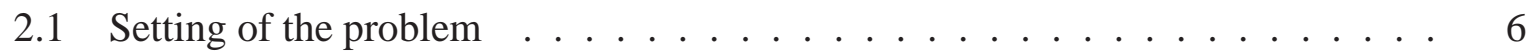

2.2 Unfolding a curve of known rotation number . . . . . . . . . . . . 8

2.3 Unfolding a curve of unknown rotation number . . . . . . . . . . . . 10

2.4 First approximation of the rotation number . . . . . . . . . . . . . . . . . 12

2.5 Computation of rotation numbers and derivatives . . . . . . . . . . . 15

2.6 Higher order unfolding of curves . . . . . . . . . . . . . . . . . . . . . . . . . . . . . . . . . . . . . . .

2.7 Extrapolation of Fourier coefficients . . . . . . . . . . . . . . . . 22

3 Some numerical illustrations $\quad 23$

3.1 Siegel domain of a quadratic polynomial . . . . . . . . . . . . . . . . 25

3.2 Study of a quasi-periodic signal . . . . . . . . . . . . . . . . 27

3.3 Study of invariant curves in non-twist maps . . . . . . . . . . . . . 30

$\begin{array}{ll}\text { Acknowledgements } & 41\end{array}$

$\begin{array}{ll}\text { References } & 41\end{array}$

\section{Introduction}

In this paper we present numerical algorithms to deal with quasi-periodic invariant curves of planar maps by adapting a method presented in [29] to compute rotation numbers of analytic circle diffeomorphisms. The developed ideas do not require the curve to be invariant under any map so they can be applied to more general objects that we refer to as quasi-periodic signals (see Definition 2.2).

The method of [29] is built assuming that the circle map is analytically ${ }^{1}$ conjugate to a rigid rotation and, basically, it consists in computing suitable averages of the iterates of the map followed by Richardson extrapolation. Since this construction takes advantage of the geometry and the dynamics of the problem, the method turns out to be highly accurate and very efficient in multiple applications. In a few words, if we compute $N$ iterates of the map, then we can approximate the rotation number with an error of order $\mathcal{O}\left(1 / N^{p+1}\right)$ where $p$ is the selected order of averaging (compared with $\mathcal{O}(1 / N)$ obtained using the definition). This methodology has been extended in [23] to deal with derivatives of the rotation number with respect to parameters. In this case, it is required to compute and average the corresponding derivatives of the iterates

\footnotetext{
${ }^{1}$ The methods of $[23,29]$ also work in the class of $\mathcal{C}^{r}$ circle diffeomorphisms, $r$ being sufficiently large, but we restrict the discussion to the analytic case in order to simplify the exposition.
} 
of the circle map. We want to point out that this variational information cannot be obtained in such a direct way by means of other existing methods to compute rotation numbers (we refer to the works $[3,4,8,15,22,36])$.

As a matter of motivation, let us assume first that $F$ is a map on the real annulus $\mathbb{T} \times I$, where $I$ is a real interval and $\mathbb{T}=\mathbb{R} / \mathbb{Z}$, and let $X: \mathbb{T} \times I \rightarrow \mathbb{R}$ denote the canonical projection $X(x, y)=x$. If $F$ is a twist ${ }^{2}$ map, the Birkhoff Graph Theorem (see [12]) ensures that every invariant curve $\Gamma$ is a graph over its projection on the circle by means of $X$, and its dynamics induces a circle map by projecting the iterates. Hence, it is straightforward to apply the method of [29] in order to approximate the rotation number of $\Gamma$, since for any $\left(x_{0}, y_{0}\right) \in \Gamma$ we can compute the orbit $x_{n}=X\left(F^{n}\left(x_{0}, y_{0}\right)\right)$-this is the only data that the method requires. Furthermore, if $F$ has a differentiable family of invariant curves or a Cantorian family differentiable in the sense of Whitney, we can approximate derivatives of the rotation number with respect to initial conditions and parameters. This allows to implement a Newton scheme for the computation and continuation of invariant curves of twist maps (as it is discussed in detail in [23]).

If the map does not satisfy the twist condition or it is not written in suitable coordinates, its invariant curves are not necessarily graphs over the projection on a circle. In this situation, invariant curves can fold in a very wild way (see Section 3.3 and references given therein for examples of such curves). Nevertheless, if we can select a suitable circle so that the folded curve "rotates" around it, then the projection of the iterates of the map does not define a circle map but a "circle correspondence" and we can compute the rotation number of the curve from the "lift" of this correspondence to the real line - see Section 2.1 for details. Moreover, albeit we do not have a justification of this fact, we realize that the extrapolation methods of [23, 29] work quite well when applied to the iterates of this "lift".

In some cases - for example, if the rotation number is large compared with the size of the folds - we can compute numerically this "lift" from the iterates of the map. However, if the curve is extremely folded additional work is required in order to face the problem in a systematic way. Hence, we propose a numerical method to construct a circle map — preserving the rotation number - from a general invariant curve on the plane. The method consists in averaging the iterates of an orbit of the curve in such a way that the new iterates belong to another curve, no longer invariant under the map, but having the same rotation number. Concretely, if we know an approximation of the rotation number with error $\varepsilon$, we construct a sequence of (averaged) curves that approaches a circle up to terms of order $\mathcal{O}(\varepsilon)$. We refer to this construction as unfolding of the curve since if $\varepsilon$ is small enough, then this construction provides us with a circle map. Taking into account the discussion in the previous paragraph, in order to apply the methods of $[23,29]$ it is not necessary to unfold completely the curve, but only to "kill" its main folds so we can compute the "lift" of the correspondence generated by the projection of the iterates of the new (less-folded) curve. In order to justify this unfolding procedure we require the curve to be analytic (or at least differentiable enough) and the rotation number to be Diophantine.

\footnotetext{
${ }^{2}$ The map $F$ satisfies the twist condition if $\partial(X \circ F) / \partial y$ does not vanishes.
} 
Sometimes the requested approximation of the rotation number is given by the context of the problem - for example, if we look for invariant curves of fixed rotation number - or it can be obtained by means of any method of frequency analysis (see for example [15, 22]). Therefore, we obtain a very efficient toolkit for the study of invariant curves of planar maps and their numerical continuation.

Let us remark that due to the importance, both theoretical and applied, of invariant curves of maps or 2-dimensional tori of flows (for example, they play a fundamental role in the design of space missions [13,14] and also in the study of models in Celestial Mechanics [32], Molecular Dynamics [27, 35] or Plasma-Beam Physics [24], just to say a few), several approaches to deal with these objects have been developed in the literature. For example, the methods in $[5,7,19]$ have been applied efficiently in a wide set of contexts. However, they require to compute a representation - by means of a trigonometric polynomial - of the curve which solves the invariance equation of the problem, so it is required to solve large systems of equations —as large as the used number of Fourier modes, say $M$. One possibility to face this difficulty is to solve these full linear systems, with a cost $\mathcal{O}\left(M^{3}\right)$ in time and $\mathcal{O}\left(M^{2}\right)$ in memory, by means of efficient parallel algorithms as it is proposed in [19]. Another recent approach presented in [7], based on the analytic and geometric ideas developed in [6], allows to reduce the computational effort of the problem to a cost of order $\mathcal{O}(M \log M)$ in time and $\mathcal{O}(M)$ in memory. On the other hand, we can compute the invariant curve by looking for a point so that the corresponding orbit has a prefixed rotation number. Then, rather than approximating explicitly the parameterization of the curve, we reduce the problem to finding a zero of a function. This approach can be implemented using interpolation methods as in [33] or also using the extrapolation methods in $[23,29]$. These extrapolation methods, that are the cornerstone of the presented paper, have a cost of order $\mathcal{O}(N \log N)$ in terms of the used number of iterates $N$ and are free in memory. Once we know a point on the curve and its rotation number, we can compute a trigonometric approximation of the curve "a posteriori", using Fourier Transform (FT) on the iterates of the curve. In addition, in Section 2.7 we develop a method for performing this FT based also on averaging-extrapolation ideas.

Given a numerical method for the continuation of invariant curves, it is specially interesting to verify if the method is valid up to the breakdown threshold corresponding to the critical invariant curve (see $[9,16,26])$. These critical curves are specially important objects that organize the long-term behavior of a given dynamical system, because of their role as "last barriers" or "bottlenecks" to chaos (see [12]). Actually, the critical value for the breakdown of the golden curve for the Chirikov standard map was estimated by means of extrapolation methods in [29] obtaining a good agreement with the value predicted by means of the classical Greene's criterion in [16]. For the non-twist case, we refer to computations in $[1,10]$ as examples of break-down studies in non-twist maps. It is worth mentioning that the methods presented in this paper can be applied also in this context.

Since our construction does not use the invariance of the curve under the map, it can be applied to the study of quasi-periodic curves that are not necessarily embedded (that we call 
quasi-periodic signals). This context is very interesting since it allows to analyze sets of data obtained from real experiments or observed natural phenomena. Actually, in order to check that the methods are robust when facing experimental data, we consider the effect of Gaussian error in the evaluation of iterates of a known quasi-periodic function.

We want to point out that our approach can be also understood as a method for the refinement of the frequency analysis of [22]. Actually, an efficient refinement of these methods, based in the simultaneous improvement of the frequencies and the amplitudes of the signal, is given in [15]. Once again, the main advantage of our approach is that we do not have to compute Fourier coefficients of the curve. This fact reduces the computational effort of solving big linear systems of equations required to refine the representation of the signal. In addition, the accuracy in the computation of the rotation number is not limited by the truncation error in the representation of the signal.

Finally, we notice that the methodology of $[23,29]$ also works for dealing with maps of the $d$-dimensional torus that admit an analytic conjugation to a rigid rotation having a Diophantine rotation vector. Our aim is to explore the extension of the ideas presented in this paper to deal with invariant tori and quasi-periodic signals of arbitrary number of frequencies.

The paper is organized as follows. In the first part, contained in Section 2, we develop and justify different results, methods and algorithms to study quasi-periodic invariant curves (or quasi-periodic signals). In the second part, presented in Section 3, we consider several examples in order to illustrate different features of the presented methodology. These examples have been selected in order to sustain the presentation of the methods and to highlight both some of the possibilities and limitations of our approach.

\section{Exposition of methods}

As we said in the introduction, we approach the study of quasi-periodic signals by computing the rotation number of a circle map (or a circle correspondence) induced by the curve. The main definitions and notation, together with a brief overview of the problem, are given in Section 2.1. After that, we present and justify a method to unfold a quasi-periodic signal. We first assume in Section 2.2 that the rotation number is known exactly in order to highlight the involved ideas. Basically, we construct a sequence of curves that converges to a circle whose dynamics corresponds to a rigid rotation. In Section 2.3 we assume that we only have an approximation of the rotation number and we show that the previous construction allows to obtain a curve that is $\mathcal{C}^{1}$-close to be a circle - the proximity being of the same order as the error in the initial guess of the rotation number.

In order to obtain the required approximation, a possibility is to resort to frequency analysis methods. In Section 2.4 we review Laskar's frequency analysis method in terms of the language presented in this paper, just to stress that the same algorithms derived to unfold the curve can be adapted to obtain the required approximation of the rotation number as alternative of the classical methods. 
For the sake of completeness we include in Section 2.5 a brief survey of the methods of [23, 29 ] to compute the rotation number and derivatives with respect to parameters of the obtained circle map or correspondence. This review is necessary to understand the higher order method that we develop in Section 2.6 to improve the unfolding of curves. During the exposition it will be clear that the ideas used in the unfolding are related to FT. This fact is exploited in Section 2.7 in order to extrapolate Fourier coefficients once the rotation number is known.

\subsection{Setting of the problem}

For convenience, we identify the real plane with the set of complex numbers by defining $z=u+\mathrm{i} v$ for any $(u, v) \in \mathbb{R}^{2}$. Let $\Gamma \subset \mathbb{C}$ be a quasi-periodic invariant curve for a map $F: U \subset \mathbb{C} \rightarrow \mathbb{C}$ of rotation number $\theta \in \mathbb{R} \backslash \mathbb{Q}$. Let us assume, for example, that the curve "rotates" around the origin and that it is a graph of the angular variable. Then, the projection

$$
\begin{aligned}
\Gamma & \longrightarrow \mathbb{T} \\
z & \longmapsto x=\arg (z) / 2 \pi
\end{aligned}
$$

generates a circle map induced by the dynamics of $\left.F\right|_{\Gamma}$. On the other hand, if $\Gamma$ is folded, then the projection (1) does not provide a circle map, but defines a correspondence on $\mathbb{T}$ that we can "lift" to $\mathbb{R}$. For example, in the left plot of Figure 1 we show a "folded" invariant curve on the complex plane for an example considered in Section 3.3. In the right plot of Figure 1 we show the "lift" of the correspondence on $\mathbb{T}$ given by (1). Since the rotation number of the curve is no more than the averaged number of revolutions per iterate, it is not surprising that we can compute it as $\lim _{n \rightarrow \infty}\left(x_{n}-x_{0}\right) / n$, where $x_{n}$ are the iterates under the "lift" to $\mathbb{R}$ of the circle correspondence. In this situation, we have observed that the methods of [23, 29] can be applied to such a "lift" (see examples in Section 3.3), even though we do not have a justification of this fact.

In some cases, for example if the rotation number is large enough as to avoid the folds, we can compute numerically the "lift" of (1) using the iterates of an orbit. However, if the invariant curve presents large folds or we cannot identify directly a good point around which the curve is rotating, we cannot compute this "lift" in a systematic way. Then, our aim is to construct another curve, having the same rotation number, by using suitable averages of iterates of the original map. If we manage to eliminate (or at least minimize) the folds in the new curve, then we are able to obtain a circle diffeomorphism (or at least a circle correspondence that we can "lift" numerically).

As $\Gamma$ is a quasi-periodic invariant curve of rotation number $\theta$, there exists an analytic embedding $\gamma: \mathbb{T} \rightarrow \mathbb{C}$ verifying $\Gamma=\gamma(\mathbb{T})$ and

$$
F(\gamma(x))=\gamma(x+\theta) .
$$

In this situation, since the parameterization $\gamma$ is periodic, we can use the Fourier series

$$
\gamma(x)=\sum_{k \in \mathbb{Z}} \hat{\gamma}_{k} \mathrm{e}^{2 \pi \mathrm{i} k x}
$$



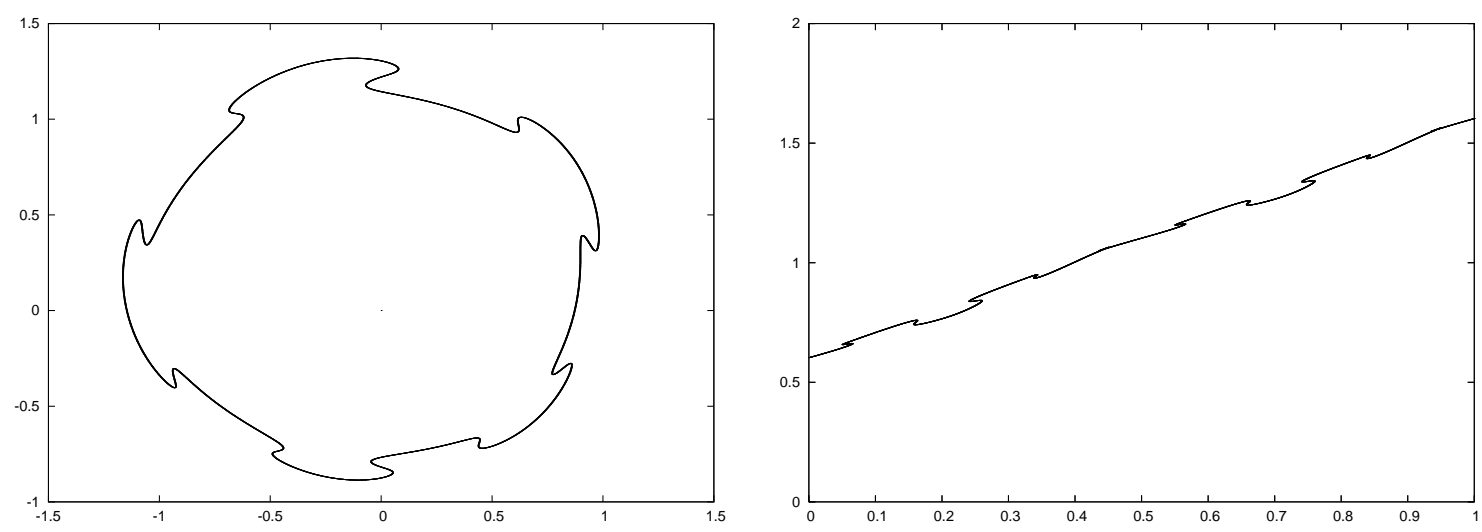

Figure 1: Left: Folded invariant curve with quasi-periodic dynamics that rotates around the origin in the complex plane (this curve corresponds to an example discussed in Section 3.3). Right: "Lift" of the associated circle correspondence given by (1).

and, moreover, for a given $z_{0} \in \Gamma$ we can ask for $\gamma(0)=z_{0}$. Then, the iterates of $z_{0}$ under $F$ can be expressed using $\gamma$ as

$$
z_{n}=F^{n}\left(z_{0}\right)=F^{n}(\gamma(0))=F^{n-1}(\gamma(\theta))=\gamma(n \theta)=\sum_{k \in \mathbb{Z}} \hat{\gamma}_{k} \mathrm{e}^{2 \pi \mathrm{i} k n \theta}
$$

As we will see, our method does not use the invariance of $\Gamma$ under $F$ but only the expression (2) for the iterates. Furthermore, even if we start with an invariant curve of a map, the intermediate stages of our construction may produce curves that are not embedded in $\mathbb{C}$. Using this fact as a motivation, we state the following definitions:

Definition 2.1. We say that a complex sequence $\left\{z_{n}\right\}_{n \in \mathbb{Z}}$ is a quasi-periodic signal of rotation number $\theta$ if there exists a periodic function $\gamma: \mathbb{T} \rightarrow \mathbb{C}$ such that $z_{n}=\gamma(n \theta)$. We also call $\Gamma=\gamma(\mathbb{T})$ a quasi-periodic curve.

Definition 2.2. Under the above conditions, let $\left\{z_{n}\right\}_{n \in \mathbb{Z}}$ be a quasi-periodic signal. Then, for any $\theta_{0} \in \mathbb{R}$ and $L \in \mathbb{N}$, we define the following iterates

$$
z_{n}^{\left(L, \theta_{0}\right)}=\frac{1}{L} \sum_{m=n}^{L+n-1} z_{m} \mathrm{e}^{2 \pi \mathrm{i}(n-m) \theta_{0}} .
$$

It is clear that $\left\{z_{n}^{\left(L, \theta_{0}\right)}\right\}_{n \in \mathbb{Z}}$ is a quasi-periodic signal on another curve $\Gamma^{\left(L, \theta_{0}\right)}=\gamma^{\left(L, \theta_{0}\right)}(\mathbb{T})$ of the same rotation number, i.e.,

$$
z_{n}^{\left(L, \theta_{0}\right)}=\gamma^{\left(L, \theta_{0}\right)}(n \theta)=\sum_{k \in \mathbb{Z}} \hat{\gamma}_{k}^{\left(L, \theta_{0}\right)} \mathrm{e}^{2 \pi \mathrm{i} k n \theta},
$$


and the new Fourier coefficients are given by

$$
\hat{\gamma}_{k}^{\left(L, \theta_{0}\right)}=\frac{\hat{\gamma}_{k}}{L} \sum_{m=n}^{L+n-1} \mathrm{e}^{2 \pi \mathrm{i}(m-n)\left(k \theta-\theta_{0}\right)}=\frac{\hat{\gamma}_{k}}{L} \frac{1-\mathrm{e}^{2 \pi \mathrm{i} L\left(k \theta-\theta_{0}\right)}}{1-\mathrm{e}^{2 \pi \mathrm{i}\left(k \theta-\theta_{0}\right)}},
$$

In Section 2.2 we show that, under conditions on regularity and non-resonance, if $\theta_{0}=\theta$, then the new curve $\gamma^{(L, \theta)}$ is arbitrarily $\mathcal{C}^{1}$-close to a circle (see Lemma 2.6) for $L$ large enough. On the other hand, if $\varepsilon=\theta_{0}-\theta$ is small, then we can choose $L=L(\varepsilon)$ such that the new curve is $\mathcal{C}^{1}$-close to a circle with an error of order $\mathcal{O}(\varepsilon)$ (this is concluded from Proposition 2.8) so that the projection

$$
\begin{aligned}
\Gamma^{\left(L, \theta_{0}\right)} \subset \mathbb{C}^{*} & \longrightarrow \mathbb{T} \\
z_{n}^{\left(L, \theta_{0}\right)} & \longmapsto x_{n}^{\left(L, \theta_{0}\right)}=\arg \left(z_{n}^{\left(L, \theta_{0}\right)}\right) / 2 \pi,
\end{aligned}
$$

provides an orbit of a circle diffeomorphism $f_{\Gamma}^{\left(L, \theta_{0}\right)}$. Once this circle map has been obtained (as we have discussed, in practice it suffices to obtain a slightly folded curve such that we can compute the "lift" of the circle correspondence defined by the direct projection), we can apply the methodology of $[23,29]$ to compute the rotation number and derivatives with respect to parameters (this is described in Secion 2.5). In order to justify this construction, we require the rotation number to be Diophantine.

Definition 2.3. Given $\theta \in \mathbb{R}$, we say that $\theta$ is a Diophantine number of $(C, \tau)$ type if there exist constants $C>0$ and $\tau \geq 1$ such that

$$
|k \theta-l|^{-1} \leq C|k|^{\tau}, \quad \forall(l, k) \in \mathbb{Z} \times \mathbb{Z}_{*}
$$

We will denote $\mathcal{D}(C, \tau)$ the set of such numbers and $\mathcal{D}$ the set of Diophantine numbers of any type.

In the aim of KAM theory, we know that the hypothesis of Diophantine rotation number for the dynamics on the curve is consistent with its own existence. Although Diophantine sets are Cantorian - i.e., compact, perfect and nowhere dense- a remarkable property is that $\mathbb{R} \backslash \mathcal{D}$ has zero Lebesgue measure. For this reason, this condition fits very well in practical issues and we do not resort to other weaker conditions on small divisors such as the Brjuno condition (see [38]). It is worth mentioning that if $\theta$ is a "bad" Diophantine rotation number, i.e., having a large constant $C$ in (7), then the methods presented in this paper turn out to be less efficient as we discuss in Section 3.

\subsection{Unfolding a curve of known rotation number}

Let us consider the previous setting and suppose that the Fourier coefficients of $\gamma$ are $\hat{\gamma}_{1} \neq 0$ and $\hat{\gamma}_{k}=0$ for $k \in \mathbb{Z} \backslash\{1\}$. In this case, $\Gamma=\left\{z \in \mathbb{C}|| z|=| \hat{\gamma}_{1} \mid\right\}$ and the circle map obtained by the projection (1) is a rigid rotation $R_{\theta}(x)=x+\theta$. 
Assume now that $\hat{\gamma}_{1} \neq 0$ and $\left|\hat{\gamma}_{k}\right|$ is small (compared with $\left|\hat{\gamma}_{1}\right|$ ) for every $k \in \mathbb{Z} \backslash\{1\}$ in such a way that the curve $\gamma$ is also $\mathcal{C}^{1}$-close to a circle. In this case, the projection $x=\arg (z) / 2 \pi$ also makes sense and defines a circle diffeomorphism.

In the general case this projection does not provide a circle map. However, it turns out that the projection of the iterates $z_{n}^{\left(L, \theta_{0}\right)}$ is well posed if we take $\theta_{0}=\theta$ and $L$ large enough. More quantitatively, we assert that the curve $\gamma^{(L, \theta)}$ differs from a circle by an amount of order $\mathcal{O}(1 / L)$. In Lemma 2.6 bellow we make precise the above arguments.

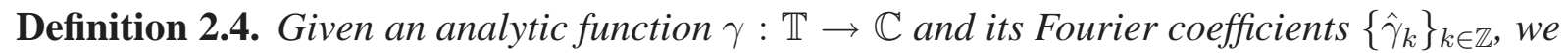
consider the norm $\|\gamma\|=\sum_{k \in \mathbb{Z}}\left|\hat{\gamma}_{k}\right|$.

Definition 2.5. Given $k \in \mathbb{Z} \backslash\{0\}$ and $r \in \mathbb{C}$, we define the map $\gamma_{k}[r]: \mathbb{T} \rightarrow \mathbb{C}$ as

$$
\gamma_{k}[r](x)=r \mathrm{e}^{2 \pi \mathrm{i} k x}
$$

Then we state the following result:

Lemma 2.6. Let us consider a quasi-periodic signal $z_{n}=\gamma(n \theta)$ of rotation number $\theta \in$ $\mathcal{D}(C, \tau)$. Assume that $\gamma: \mathbb{T} \rightarrow \mathbb{C}$ is analytic in the complex strip $B_{\Delta}=\{z \in \mathbb{C}:|\operatorname{Im}(z)|<\Delta\}$ and bounded in the closure, with $M=\sup _{z \in B_{\Delta}}|\gamma(z)|$. Then, if $\hat{\gamma}_{1} \neq 0$, the curve $\gamma^{(L, \theta)}: \mathbb{T} \rightarrow$ $\mathbb{C}$ given by (4) and (5) satisfies

$$
\left\|\gamma^{(L, \theta)}-\gamma_{1}\left[\hat{\gamma}_{1}\right]\right\| \leq \frac{A}{L}
$$

where $A$ is a constant depending on $M, C, \tau$ and $\Delta$.

Proof. First, let us observe that the Fourier coefficients of the new curve are given by

$$
\hat{\gamma}_{1}^{(L, \theta)}=\hat{\gamma}_{1}, \quad \hat{\gamma}_{k}^{(L, \theta)}=\frac{\hat{\gamma}_{k}}{L} \frac{1-\mathrm{e}^{2 \pi \mathrm{i}(k-1) \theta L}}{1-\mathrm{e}^{2 \pi \mathrm{i}(k-1) \theta}}, \quad k \in \mathbb{Z} \backslash\{1\} .
$$

Then, we have to bound the expression

$$
\left\|\gamma^{\left(L, \theta_{0}\right)}-\gamma_{1}\left[\hat{\gamma}_{1}\right]\right\| \leq \frac{1}{L} \sum_{k \in \mathbb{Z} \backslash\{1\}}\left|\hat{\gamma}_{k} \frac{1-\mathrm{e}^{2 \pi \mathrm{i}(k-1) \theta L}}{1-\mathrm{e}^{2 \pi \mathrm{i}(k-1) \theta}}\right| \leq \frac{1}{L} \sum_{k \in \mathbb{Z} \backslash\{1\}} \frac{2\left|\hat{\gamma}_{k}\right|}{\left|1-\mathrm{e}^{2 \pi \mathrm{i}(k-1) \theta}\right|} .
$$

We observe that the Fourier coefficients of $\gamma$ satisfy $\left|\hat{\gamma}_{k}\right| \leq M \mathrm{e}^{-2 \pi \Delta|k|}$ and we use (7) to control the small divisors. Concretely, standard manipulations show that (see for example [2])

$$
\left|1-\mathrm{e}^{2 \pi \mathrm{i}(k-1) \theta}\right|^{-1} \leq \frac{C}{4}|k-1|^{\tau} .
$$

Introducing these expressions in the previous sum we obtain that

$$
\left\|\gamma^{\left(L, \theta_{0}\right)}-\gamma_{1}\left[\hat{\gamma}_{1}\right]\right\| \leq \frac{C M}{2 L} \sum_{k \in \mathbb{Z} \backslash\{1\}}|k-1|^{\tau} \mathrm{e}^{-2 \pi \Delta|k|} \leq \frac{C M}{L} \sup _{x \geq 0}\left\{\mathrm{e}^{-\pi \Delta x}(x+1)^{\tau}\right\} \sum_{k=0}^{\infty} \mathrm{e}^{-\pi \Delta k} .
$$


Moreover, we observe that

$$
\sup _{x \geq 0}\left\{\mathrm{e}^{-s x}(x+1)^{m}\right\}=\left\{\begin{array}{cl}
1 & \text { if } s \geq m \\
(m /(s \mathrm{e}))^{m} \mathrm{e}^{s} & \text { if } s<m .
\end{array}\right.
$$

Finally, taking $A=\frac{M C}{1-\mathrm{e}^{-\pi \Delta}}\left(1+\left(\frac{\tau}{\pi \Delta}\right)^{\tau}\right)$ the stated bound follows immediately.

Remark 2.7. Note that in order to guarantee that the projection (6) is well posed we also need to control the derivative $\left(\gamma^{(L, \theta)}\right)^{\prime}(x)$. Of course, this can be done modifying slightly the proof of Lemma 2.6.

\subsection{Unfolding a curve of unknown rotation number}

Since we are concerned with the computation of $\theta$, the construction presented in the previous section seems useless. Next we show that the method still works - with certain restrictionsif the rotation number $\theta$ is unknown, but we have an approximation $\theta_{0}$.

Proposition 2.8. Let us consider a quasi-periodic signal $z_{n}=\gamma(n \theta)$ of rotation number $\theta \in$ $\mathcal{D}(C, \tau)$. Assume that $\gamma: \mathbb{T} \rightarrow \mathbb{C}$ is analytic in the complex strip $B_{\Delta}$ and bounded in the closure, with $M=\sup _{z \in B_{\Delta}}|\gamma(z)|$. Suppose that $\theta_{0}$ is an approximation of $\theta$ and let us denote $\varepsilon=\theta_{0}-\theta$ and $K_{\varepsilon}=\left\lfloor(2 C|\varepsilon|)^{-1 / \tau}\right\rfloor$. Then, if $\hat{\gamma}_{1} \neq 0$ and $K_{\varepsilon} \geq 1$, for every $L \in \mathbb{N}$ the following estimate holds

$$
\frac{\left\|\gamma^{\left(L, \theta_{0}\right)}-\gamma_{1}\left[\hat{\gamma}_{1}^{\left(L, \theta_{0}\right)}\right]\right\|}{\left|\hat{\gamma}_{1}^{\left(L, \theta_{0}\right)}\right|} \leq\left|\frac{\sin (\pi \varepsilon)}{\sin (\pi \varepsilon L)}\right|\left(\frac{A}{\left|\hat{\gamma}_{1}\right|}+\frac{2 M L}{\left|\hat{\gamma}_{1}\right|} \frac{\mathrm{e}^{-2 \pi \Delta\left(K_{\varepsilon}-1\right)}}{1-\mathrm{e}^{-2 \pi \Delta}}\right),
$$

where $A$ is a constant depending on $M, C, \tau$ and $\Delta$.

Proof. Let us consider the sets

$$
\begin{aligned}
K(\varepsilon) & =\left\{k \in \mathbb{Z} \backslash\{1\}:|k-1| \leq K_{\varepsilon}\right\}, \\
K^{*}(\varepsilon) & =\mathbb{Z} \backslash(K(\varepsilon) \cup\{1\}) .
\end{aligned}
$$

Then, if $k \in K(\varepsilon)$ the following bound is satisfied $\forall l \in \mathbb{Z}$

$$
\left|k \theta-\theta_{0}-l\right| \geq|(k-1) \theta-l|-|\varepsilon| \geq \frac{1}{C|k-1|^{\tau}}-|\varepsilon| \geq \frac{1}{2 C|k-1|^{\tau}}
$$

allowing to control the small divisors

$$
\left|1-\mathrm{e}^{2 \pi \mathrm{i}\left(k \theta-\theta_{0}\right)}\right| \geq \frac{2}{C|k-1|^{\tau}} \quad \forall k \in K(\varepsilon) .
$$

Then, from formula (5) and recalling that the Fourier coefficients satisfy $\left|\hat{\gamma}_{k}\right| \leq M \mathrm{e}^{-2 \pi \Delta|k|}$, we obtain estimates for $\hat{\gamma}_{k}^{\left(L, \theta_{0}\right)}$. If $k \in K(\varepsilon)$, we use the expression (9) to obtain $\left|\hat{\gamma}_{k}^{\left(L, \theta_{0}\right)}\right| \leq$ 
$M C L^{-1}|k-1|^{\tau} \mathrm{e}^{-2 \pi \Delta|k|}$. On the other hand, for indexes $k \in K^{*}(\varepsilon)$ we use that $\left|\hat{\gamma}_{k}^{\left(L, \theta_{0}\right)}\right| \leq\left|\hat{\gamma}_{k}\right|$. Therefore, we have to consider the following sums

$$
\left\|\gamma^{\left(L, \theta_{0}\right)}-\gamma_{1}\left[\hat{\gamma}_{1}^{\left(L, \theta_{0}\right)}\right]\right\| \leq \frac{M C}{L} \sum_{k \in K(\varepsilon)}|k-1|^{\tau} \mathrm{e}^{-2 \pi \Delta|k|}+M \sum_{k \in K^{*}(\varepsilon)} \mathrm{e}^{-2 \pi \Delta|k|} .
$$

Now, the sum for $k \in K(\varepsilon)$ is controlled by splitting it into the sets $\left[-K_{\varepsilon}+1,0\right] \cap \mathbb{Z}$ and $\left[2, K_{\varepsilon}+1\right] \cap \mathbb{Z}$. Then, we proceed as in the proof of Lemma 2.6 obtaining the constant $A=\frac{2 M C}{1-\mathrm{e}^{-\pi \Delta}}\left(1+\left(\frac{\tau}{\pi \Delta}\right)^{\tau}\right)$. Finally, we compute the first Fourier coefficient

$$
\left|\hat{\gamma}_{1}^{\left(L, \theta_{0}\right)}\right|=\left|\frac{\hat{\gamma}_{1}}{L}\right|\left|\frac{1-\mathrm{e}^{-2 \pi \mathrm{i} \varepsilon L}}{1-\mathrm{e}^{-2 \pi \mathrm{i} \varepsilon}}\right|=\left|\frac{\hat{\gamma}_{1}}{L}\right|\left|\frac{\sin (\pi \varepsilon L)}{\sin (\pi \varepsilon)}\right|,
$$

ending up with estimate (8).

Remark 2.9. If we restrict Lemma 2.8 to those values of $\theta_{0}$ such that the Diophantine condition (9) is valid $\forall k \in \mathbb{Z} \backslash\{1\}$, then we obtain the estimate

$$
\frac{\left\|\gamma^{\left(L, \theta_{0}\right)}-\gamma_{1}\left[\hat{\gamma}_{1}^{\left(L, \theta_{0}\right)}\right]\right\|}{\left|\hat{\gamma}_{1}^{\left(L, \theta_{0}\right)}\right|} \leq\left|\frac{\sin (\pi \varepsilon)}{\sin (\pi \varepsilon L)}\right| \frac{A}{\left|\hat{\gamma}_{1}\right|} .
$$

Indeed, if we denote $\mathcal{E} \subset \mathbb{R}$ the set of values of $\varepsilon$ such that $\theta_{0}=\theta+\varepsilon$ satisfies estimate (9) for every $k \in \mathbb{Z} \backslash\{1\}$, then for every $\varepsilon_{0}$ sufficiently small the measure of the set $\left[-\varepsilon_{0}, \varepsilon_{0}\right] \backslash \mathcal{E}$ is exponentially small in $\varepsilon_{0}{ }^{3}$

Observe that for any fixed $|\varepsilon|>0$, estimate (10) depends $\left|\frac{1}{\varepsilon}\right|$-periodically on $L$ and also does (8) modulo exponentially small terms in $|\varepsilon|$. Since we are interested in the minimization of (8), we point out that if $L \simeq\left|\frac{1}{2 \varepsilon}\right|$, then

$$
\frac{\left\|\gamma^{\left(L, \theta_{0}\right)}-\gamma_{1}\left[\hat{\gamma}_{1}^{\left(L, \theta_{0}\right)}\right]\right\|}{\left|\hat{\gamma}_{1}^{\left(L, \theta_{0}\right)}\right|}=\mathcal{O}(\varepsilon) .
$$

Hence, the new parameterization is closer to a circle for $|\varepsilon|$ sufficiently small and the projection

$$
\begin{aligned}
\Gamma^{\left(L, \theta_{0}\right)} \subset \mathbb{C}^{*} & \longrightarrow \mathbb{T} \\
z_{n}^{\left(L, \theta_{0}\right)} & \longmapsto x_{n}^{\left(L, \theta_{0}\right)}=\arg \left(z_{n}^{\left(L, \theta_{0}\right)}\right) / 2 \pi,
\end{aligned}
$$

induces a well-posed circle diffeomorphism that we denote as $f_{\Gamma}^{\left(L, \theta_{0}\right)}$. Of course, the regularity of the circle map $f_{\Gamma}^{\left(L, \theta_{0}\right)}$ follows from the regularity of $\gamma$. Hence, we can compute the rotation number $\theta$ and derivatives with respect to parameters by applying the methods of $[23,29]$ that we recall briefly in Section 2.5. Before that, we discuss how this required guess $\theta_{0}$ can be obtained.

\footnotetext{
${ }^{3}$ These two points of view are analogous to different approaches followed in [20] and [21] to study reducibility of quasi-periodic linear equations.
} 


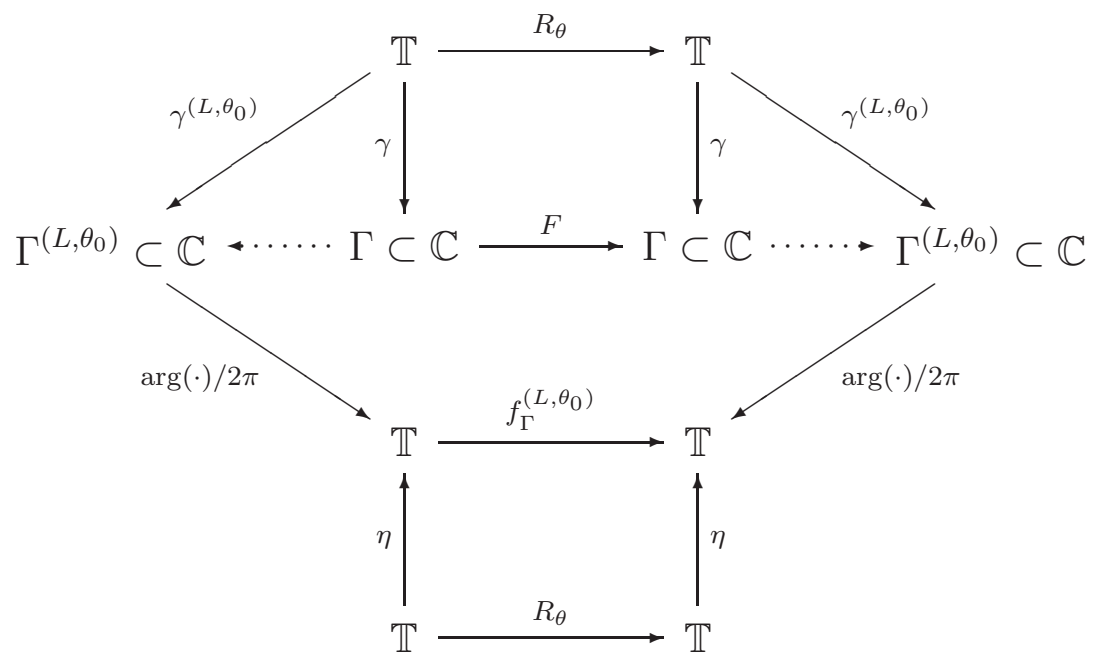

Figure 2: This diagram summarizes the construction of the analytic circle diffeomorphism $f_{\Gamma}^{\left(L, \theta_{0}\right)}$ from a folded invariant curve $\Gamma$ of $F$ of rotation number $\theta$.

\subsection{First approximation of the rotation number}

The classical frequency analysis approach introduced by J. Laskar (see [22]) to obtain an approximation of frequencies of a quasi-periodic signal - here we are considering only one independent frequency - is to look for the frequencies as peaks of the modulus of the Discrete Fourier Transform (DFT) of the studied signal. In this section we translate the elementary ideas used in frequency analysis into the terminology introduced in Section 2.3.

Let us focus on the iterates $\left\{z_{n}^{\left(L, \theta_{0}\right)}\right\}_{n \in \mathbb{Z}}$ of Definition 2.2. We observe that they look very similar to the DFT of the signal. We also notice that they can be defined for any $\theta_{0}$ but the Fourier coefficient $\hat{\gamma}_{1}^{\left(L, \theta_{0}\right)}$, given explicitly in (5), has a local maximum when $\theta_{0}$ equals $\theta$, and from Proposition 2.8 we conclude that, for $L$ large enough, the function $\theta_{0} \mapsto\left|z_{n}^{\left(L, \theta_{0}\right)}\right|$ has a local maximum for a value of $\theta_{0}$ close to $\theta$. In general, this phenomena occurs for the $t$ th coefficient if we select $\theta_{0}$ close to an integer multiple of the rotation number, i.e., $\theta_{0}=t \theta+\varepsilon, t \in \mathbb{Z}$. The corresponding justification is given by the following proposition (the proof is analogous to that of Proposition 2.8).

Proposition 2.10. Let us consider a quasi-periodic signal $z_{n}=\gamma(n \theta)$ of rotation number $\theta \in \mathcal{D}(C, \tau)$. Assume that $\gamma: \mathbb{T} \rightarrow \mathbb{C}$ is analytic in the complex strip $B_{\Delta}$ and bounded in the closure, with $M=\sup _{z \in B_{\Delta}}|\gamma(z)|$. Suppose that $\theta_{0}$ is an approximation of $t \theta$ and let us denote $\varepsilon=\theta_{0}-t \theta$ and $K_{\varepsilon}=\left\lfloor(2 C|\varepsilon|)^{-1 / \tau}\right\rfloor$. Then, if $\hat{\gamma}_{t} \neq 0$ and $K_{\varepsilon} \geq|t|$, for every $L \in \mathbb{N}$ the following estimate holds

$$
\frac{\left\|\gamma^{\left(L, \theta_{0}\right)}-\gamma_{t}\left[\hat{\gamma}_{t}^{\left(L, \theta_{0}\right)}\right]\right\|}{\left|\hat{\gamma}_{t}^{\left(L, \theta_{0}\right)}\right|} \leq\left|\frac{\sin (\pi \varepsilon)}{\sin (\pi \varepsilon L)}\right|\left(\frac{A}{\left|\hat{\gamma}_{t}\right|}+\frac{2 M L}{\left|\hat{\gamma}_{t}\right|} \frac{\mathrm{e}^{-2 \pi \Delta\left(K_{\varepsilon}-|t|\right)}}{1-\mathrm{e}^{-2 \pi \Delta}}\right),
$$


where $A=\frac{2 M C}{1-\mathrm{e}^{-\pi \Delta}}\left(|t|^{\tau}+\left(\frac{\tau}{\pi \Delta}\right)^{\tau}\right)$.

According with this result and the previous discussion, we summarize the following observations:

- First, we notice that Remark 2.9 also holds in this context, and so we conclude that this estimate behaves periodically in $L$ for most of the values of $\theta_{0}$ close to $t \theta$.

- From equation (5), we observe that $\hat{\gamma}_{t}^{\left(L, \theta_{0}\right)} \rightarrow \hat{\gamma}_{t}$ when $\varepsilon \rightarrow 0$ and that the modulus $\left|\hat{\gamma}_{t}\right|$ is an upper bound for $\left|\hat{\gamma}_{t}^{\left(L, \theta_{0}\right)}\right|$. Moreover, for $L$ sufficiently large, we obtain a local maximum in the modulus of the iterates $z_{n}^{\left(L, \theta_{0}\right)}$ for a value of $\theta_{0}$ close to $t \theta$.

- On the other hand, the estimate (11) grows with $|t|$ thus implying that, for fixed $L$, only low order harmonics can be detected.

The previous discussion gives us a heuristic method for computing an approximation $\theta_{0}$ of the rotation number $\theta$ (and its multiples modulo 1). Basically, we fix $L$ and compute the iterates $z_{n}^{\left(L, \theta_{0}\right)}$ for different values of $\theta_{0}$ in order to compute local maxima of the modulus. In particular, if we just study the modulus of the initial iterate $z_{0}^{\left(L, \theta_{0}\right)}$, we recover the method of [22]. This method is enough for our purposes - we recall that we just look for a rough approximation of the rotation number- but in Remark 2.12 we explain some refinements that can be performed in this procedure. Thus, from the method of [22] we find a finite number of candidates for the rotation number and we have to decide which one is the generator. Details are given in the next four steps.

Step 1: Maxima chasing. First, we fix $L \in \mathbb{N}$ and define the function

$$
\begin{aligned}
& \mathbb{T} \longrightarrow \mathbb{R} \\
& \theta_{0} \longmapsto\left|z_{0}^{\left(L, \theta_{0}\right)}\right|=\left|\frac{1}{L} \sum_{m=0}^{L-1} z_{m} \mathrm{e}^{-2 \pi \mathrm{i} m \theta_{0}}\right|
\end{aligned}
$$

We want to obtain values of $\theta_{0}$ that correspond to maxima of the function (12). To this end, let us consider a sample of points $\left\{\theta_{0}^{i}\right\}_{i=1, \ldots, N}$, where $N \in \mathbb{N}$ and $\theta_{0}^{i} \in[0,1]$ (actually, one can reduce the interval if some information about the rotation number is available, say $\left.\theta \in\left[\theta_{\min }, \theta_{\max }\right]\right)$. Then, for every pair $\left\{\theta_{0}^{j}, \theta_{0}^{j+1}\right\}, j=1, \ldots, N-1$, we compute a local maximum for (12) by means of golden section search using a tolerance $\varepsilon_{G S S}$ (we refer to [28] for details). Then, we introduce the following terminology:

- $\widetilde{\theta}_{0}^{j}$ : Maximum obtained from the pair $\left\{\theta_{0}^{j}, \theta_{0}^{j+1}\right\}$. Let us observe that this maximum is not necessarily contained in the interval $\left[\theta_{0}^{j}, \theta_{0}^{j+1}\right]$.

- $n_{p}$ : Number of maxima obtained at the end of this step. In order to avoid redundant information, two maxima are considered equivalent if $d_{\mathbb{T}}\left(\widetilde{\theta}_{0}^{j}, \widetilde{\theta}_{0}^{k}\right) \leq 10 \varepsilon_{G S S}$ where $d_{\mathbb{T}}$ is the quotient metric induced on the torus. 
Step 2: Maxima selection. Now we sort the obtained points $\left\{\widetilde{\theta}_{0}^{i}\right\}_{1, \ldots, n_{p}}$ according to

$$
\left|z_{0}^{\left(L, \widetilde{\theta}_{0}^{i}\right)}\right|>\left|z_{0}^{\left(L, \widetilde{\theta}_{0}^{j}\right)}\right| \quad \text { provided } \quad i<j
$$

At this point, we select the first $n_{u}$ points just omitting those ones whose corresponding maxima are small when compared with $\left|z_{0}^{\left(L, \widetilde{\theta}_{0}^{1}\right)}\right|$. In particular, we only take those elements such that

$$
\left|z_{0}^{\left(L, \widetilde{\theta}_{0}^{1}\right)}\right|<\nu\left|z_{0}^{\left(L, \widetilde{\theta}_{0}^{i}\right)}\right|
$$

where $\nu>1$ is a "selecting factor" (we typically take values of $\nu$ between 3 and 6) and we denote by $\left\{\theta_{0}^{k}\right\}_{k=1, \ldots, n_{u}}$ the set of numbers thus obtained.

Step 3: Rotation number selection. This set $\left\{\theta_{0}^{k}\right\}_{k=1, \ldots, n_{u}}$, with $\theta_{0}^{k} \in[0,1]$, corresponds to approximate multiples of the rotation number computed modulo 1. In addition, if $\left|\hat{\gamma}_{1}\right|$ is not too small, there is an element in this set that approximates the rotation number. Notice that for every $\theta_{0}^{k}$ there exist $m_{k}, n_{k} \in \mathbb{Z}$ such that $\theta_{0}^{k} \approx m_{k} \theta+n_{k}$. This motivates the following definitions

$$
\begin{array}{cc}
k_{i j}=\operatorname{argmin}_{k \in \mathbb{Z}}\left\{d_{\mathbb{T}}\left(k \theta_{0}^{i}, \theta_{0}^{j}\right)\right\}, & \kappa_{i}=\sum_{j=1}^{n_{u}}\left|k_{i j}\right| \\
d_{i j}=\min _{k \in \mathbb{Z}}\left\{d_{\mathbb{T}}\left(k \theta_{0}^{i}, \theta_{0}^{j}\right)\right\}, & \delta_{i}=\sum_{j=1}^{n_{u}} d_{i j} .
\end{array}
$$

Let us observe that if we assume that $\theta_{0}^{i} \approx \theta$, then $\kappa_{i}$ corresponds to the sum of the order of Fourier terms that allow to approximate the remaining points $\theta_{0}^{j}$. On the other hand, $\delta_{i}$ gives an idea of the error when $\theta_{0}^{i}$ is selected. If the minimum values of $\left\{\kappa_{i}\right\}_{i=1, \ldots, n_{u}}$ and $\left\{\delta_{i}\right\}_{i=1, \ldots, n_{u}}$ correspond to the same index $k \in\left\{1, \ldots, n_{u}\right\}$, then we select $\theta_{0}=\theta_{0}^{k}$ as an approximation of $\theta$. If they do not coincide but $\delta_{k}=\min _{i}\left\{\delta_{i}\right\}$ is small, then we select $\theta_{0}=\theta_{0}^{k}$. Otherwise we start again from Step 1 using a larger value of $L$.

Step 4: Validation and iteration. Of course, it is recommended to verify that the computations are stable by repeating the process (from Step 1) with larger values of $N$ and $L$.

Remark 2.11. When the frequency of the quasi-periodic signal is not an integer multiple of the "basic frequency" 1/L associated to the sample interval of the associated DFT in (12), there appear in the DFT spurious frequencies, that is, the DFT is different from zero at frequencies not being multiple of the frequency of the function. This is a phenomenon known as leakage, that it can be reduced by means of the so-called filter or window functions (see [15, 22]). Nevertheless, these spurious peaks are smaller than the corresponding harmonic that generates them, so we get rid of them in Step 2 of the described procedure. 
Remark 2.12. Finally, we notice that this procedure can be modified in several ways taking into account the ideas introduced in this paper. For example, when looking for local maxima of function (12) we can minimize with respect to $\theta_{0}$ the distance of the iterates $\left\{z_{n}^{\left(L, \theta_{0}\right)}\right\}_{n \in \mathbb{Z}}$ to be on a circle - see Proposition 2.10 - that can be measured by means of several criteria discused in Section 3.1. On the other hand, we can use higher order averages as discused in Section 2.6 in order to improve the resolution of the maxima. These refinements may become relevant when dealing with more than one frequency as a possible alternative to the filters mentioned in Remark 2.11.

\subsection{Computation of rotation numbers and derivatives}

Next, we include a brief review of the methods developed in $[23,29]$ to compute numerically the rotation number of circle diffeomorphisms together with derivatives with respect to parameters. We include this review to set the notation of the rest of the paper and also in order to remark that ideas of Section 2.6 and 2.7 follow from those introduced in [29] in a close way.

Given an orientation-preserving circle homeomorphism $f: \mathbb{T} \rightarrow \mathbb{T}$, we identify $f$ with its lift to $\mathbb{R}$ by fixing the normalization condition $f(0) \in[0,1)$. Then, we recall that the rotation number of $f$ is defined as the limit

$$
\theta=\lim _{|n| \rightarrow \infty} \frac{f^{n}\left(x_{0}\right)-x_{0}}{n}
$$

that exists for all $x_{0} \in \mathbb{R}$, is independent of $x_{0}$ and satisfies $\theta \in[0,1)$. It is well known (we refer to [17]) that if $f$ is an analytic diffeomorphism and $\theta \in \mathcal{D}$, then $f$ is analytically conjugate to a rigid rotation $R_{\theta}(x)=x+\theta$, i.e., there exists an orientation-preserving analytic circle diffeomorphism $\eta$ such that $f \circ \eta=\eta \circ R_{\theta}$. Moreover, we can write this conjugacy as $\eta(x)=x+\xi(x), \xi$ being a 1-periodic function normalized in such a way that $\xi(0)=x_{0}$, for a fixed $x_{0} \in[0,1)$. Now, by using the fact that $\eta$ conjugates $f$ to a rigid rotation, we can write the following expression for the iterates under the lift:

$$
f^{n}\left(x_{0}\right)=f^{n}(\eta(0))=\eta(n \theta)=n \theta+\sum_{k \in \mathbb{Z}} \hat{\xi}_{k} \mathrm{e}^{2 \pi \mathrm{i} k n \theta}, \quad \forall n \in \mathbb{Z},
$$

where the sequence $\left\{\hat{\xi}_{k}\right\}_{k \in \mathbb{Z}}$ denotes the Fourier coefficients of $\xi$. Then, the above expression gives us the following formula

$$
\frac{f^{n}\left(x_{0}\right)-x_{0}}{n}=\theta+\frac{1}{n} \sum_{k \in \mathbb{Z}_{*}} \hat{\xi}_{k}\left(\mathrm{e}^{2 \pi \mathrm{i} k n \theta}-1\right),
$$

to compute $\theta$ modulo terms of order $\mathcal{O}(1 / n)$. Unfortunately, this order of convergence is very slow for practical purposes, since it requires a huge number of iterates if we want to compute 
$\theta$ with high precision. Nevertheless, by averaging the iterates $f^{n}\left(x_{0}\right)$ in a suitable way, we can manage to decrease the size of the quasi-periodic remainder.

Given $p \in \mathbb{N} \cup\{0\}$, that we call the averaging order, we introduce the following recursive sums of order $p$

$$
S_{N}^{0}=f^{N}\left(x_{0}\right)-x_{0}, \quad S_{N}^{p}=\sum_{j=1}^{N} S_{j}^{p-1},
$$

and the corresponding averaged sums of order $p$

$$
\widetilde{S}_{N}^{p}=\left(\begin{array}{c}
N+p \\
p+1
\end{array}\right)^{-1} S_{N}^{p}
$$

Then, as it is shown in [29], these averages satisfy the following property.

Proposition 2.13. If $f$ is the lift of an orientation-preserving analytic circle diffeomorphism of rotation number $\theta \in \mathcal{D}$, then the following expression holds

$$
\widetilde{S}_{N}^{p}=\theta+\sum_{l=1}^{p} \frac{\hat{A}_{l}^{p}}{N^{l}}+\hat{E}^{p}(N),
$$

where the coefficients $\hat{A}_{l}^{p}$ depend on $f$ and $p$ but are independent of $N$. Furthermore, the remainder $\hat{E}^{p}(N)$ is uniformly bounded by an expression of order $\mathcal{O}\left(1 / N^{p+1}\right)$.

Let us observe that equation (17) allows to extrapolate the rotation number just by computing $\widetilde{S}_{N}^{p}$ for different values of $N$, neglecting the remainder and solving a set of linear equations.

Algorithm 2.14. Once an averaging order $p$ is selected, we take $N=2^{q}$ iterates of the map, for some $q>p$, and compute the sums $\left\{\widetilde{S}_{N_{j}}^{p}\right\}_{j=0, \ldots, p}$ with $N_{j}=2^{q-p+j}$. We approximate the rotation number using the formula

$$
\theta=\Theta_{q, p}+\mathcal{O}\left(2^{-(p+1) q}\right), \quad \Theta_{q, p}=\sum_{j=0}^{p} c_{j}^{(p)} \widetilde{S}_{2^{q-p+j}}^{p},
$$

where the coefficients $c_{j}^{(p)}$ are given by

$$
c_{l}^{(p)}=(-1)^{p-l} \frac{2^{l(l+1) / 2}}{\delta(l) \delta(p-l)},
$$

with $\delta(n)=\left(2^{n}-1\right)\left(2^{n-1}-1\right) \cdots\left(2^{1}-1\right)$ for $n \geq 1$ and $\delta(0)=1$. The operator $\Theta_{q, p}$ corresponds to the Richardson extrapolation of order $p$ of equation (17).

As far as the behavior of the error is concerned, if we fix the extrapolation order $p$ and compute $\Theta_{q, p}$, we know that $\left|\theta-\Theta_{q, p}\right| \leq c / 2^{q(p+1)}$, for certain (unknown) constant $c$ independent of $q$ (see [29]). To estimate $c$, we compute $\Theta_{q-1, p}$ and consider the expression 
$\left|\theta-\Theta_{q-1, p}\right| \leq c / 2^{(q-1)(p+1)}$. Then, we replace in this inequality the exact value of $\theta$ by $\Theta_{q, p}$, as we expect $\Theta_{q, p}$ to be closer to $\theta$ than $\Theta_{q-1, p}$. After that, we estimate $c$ by

$$
c \simeq 2^{(q-1)(p+1)}\left|\Theta_{q, p}-\Theta_{q-1, p}\right|
$$

From this approximation we obtain the following (heuristic) expression

$$
\left|\theta-\Theta_{q, p}\right| \leq \frac{\nu}{2^{p+1}}\left|\Theta_{q, p}-\Theta_{q-1, p}\right|
$$

where $\nu$ is a "safety parameter" whose role is to prevent oscillations of $c$ a function of $q$ due to the quasi-periodic part. In the computations of Section 3 we take $\nu=10$ (this value works quite well as observed in [29]).

Furthermore, let us consider a family $\mu \in I \subset \mathbb{R} \mapsto f_{\mu}$ of orientation-preserving analytic circle diffeomorphisms depending $\mathcal{C}^{d}$-smoothly with respect to $\mu$. The rotation numbers of the family $\left\{f_{\mu}\right\}_{\mu \in I}$ induce a function $\theta: I \rightarrow[0,1)$ given by $\theta(\mu)=\rho\left(f_{\mu}\right)$. Let us remark that the function $\theta$ is continuous but non-smooth: generically, there exist a family of disjoint open intervals of $I$, with dense union, such that $\theta$ takes distinct constant values on these intervals (a so-called Devil's Staircase). However, the derivatives of $\theta$ are defined in "many" points (see the discussion in [23] and references given therein).

In order to compute $D_{\mu}^{d} \theta\left(\mu_{0}\right)$, the $d$-th derivative with respect to $\mu$ at $\mu_{0}$, we proceed as before and define recursive sums of order $p$ (we omit the notation regarding the fact that the map is evaluated at $\mu=\mu_{0}$ )

$$
D_{\mu}^{d} S_{N}^{0}=D_{\mu}^{d}\left(f_{\mu}^{N}\left(x_{0}\right)-x_{0}\right), \quad D_{\mu}^{d} S_{N}^{p}=\sum_{j=0}^{N} D_{\mu}^{d} S_{j}^{p-1}
$$

and the corresponding averaged sums

$$
D_{\mu}^{d} \widetilde{S}_{N}^{p}=\left(\begin{array}{c}
N+p \\
p+1
\end{array}\right)^{-1} D_{\mu}^{d} S_{N}^{p}
$$

Proposition 2.15. If $\theta\left(\mu_{0}\right) \in \mathcal{D}$ and $D_{\mu}^{d} \theta\left(\mu_{0}\right)$ exists, we obtain (omitting the point $\mu_{0}$ )

$$
D_{\mu}^{d} \widetilde{S}_{N}^{p}=D_{\mu}^{d} \theta+\sum_{l=1}^{p-d} \frac{D_{\mu}^{d} \hat{A}_{l}^{p}}{N^{l}}+D_{\mu}^{d} \hat{E}^{p}(N),
$$

where the remainder $D_{\mu}^{d} \hat{E}^{p}(N)$ is of $\operatorname{order} \mathcal{O}\left(1 / N^{p-d+1}\right)$.

Therefore, according to formula (21), we implement the following algorithm to extrapolate the $d$-th derivative of the rotation number. 
Algorithm 2.16. Once an averaging order $p$ is selected, we take $N=2^{q}$ iterates of the map, for some $q>p$, and compute the sums $\left\{D_{\mu}^{d} \widetilde{S}_{N_{j}}^{p}\right\}_{j=0, \ldots, p}$ with $N_{j}=2^{q-p+j+d}$. We approximate the $d$-th derivative of the rotation number using the formula

$$
D_{\mu}^{d} \theta=\Theta_{q, p, p-d}^{d}+\mathcal{O}\left(2^{-(p-d+1) q}\right), \quad \Theta_{q, p, m}^{d}=\sum_{j=0}^{m} c_{j}^{(m)} D_{\mu}^{d} \widetilde{S}_{2^{q-m+j}}^{p},
$$

where the coefficients $c_{j}^{(m)}$ are also given by equation (19). The operator $\Theta_{q, p, p-d}^{d}$ corresponds to the Richardson extrapolation of order $p-d$ of equation (21).

In this case, we obtain the following heuristic expression for the extrapolation error

$$
\left|D_{\mu}^{d} \theta-\Theta_{q, p, p-d}^{d}\right| \leq \frac{\nu}{2^{p-d+1}}\left|\Theta_{q, p, p-d}^{d}-\Theta_{q-1, p, p-d}^{d}\right| .
$$

We remark that if we select an averaging order $p$, then we are limited to extrapolate with order $p-d$ instead of $p$. Moreover, $p$ is the maximum order of the derivative that can be computed.

Let us observe that, in order to approximate derivatives of the rotation number, we require to compute efficiently the quantities $D_{\mu}^{d}\left(f_{\mu}^{n}(x)\right)$, i.e., the derivatives with respect to the parameter of the iterates of an orbit. If the family $\mu \mapsto f_{\mu}$ is known explicitly or it is induced directly by a map on the annulus, several algorithms based on recursive and combinatorial formulas are detailed in [23]. In the rest of this section we develop recursive formulas to compute these derivatives when the family comes from a general planar map.

Let us consider an analytic map $F: \mathbb{C} \rightarrow \mathbb{C}$, with $F=F_{1}+\mathrm{i} F_{2}$, having a Cantor family of invariant curves differentiable in the sense of Whitney, i.e., there exists a family of parameterizations $\mu \in U \mapsto \gamma^{\mu}$ defined in a Cantor set $U$ such that $\gamma^{\mu}(\mathbb{T})=\Gamma^{\mu}$ and $F\left(\gamma^{\mu}(x)\right)=F(x+\theta(\mu))$, for $\theta(\mu) \in \mathcal{D}$. In the following, we fix a value of the parameter and we omit the dependence on $\mu$ in order to simplify the notation and we write $z_{n}=F^{n}\left(z_{0}\right)$, for $z_{0} \in \Gamma$. As in Section 2, we consider a curve $\gamma$ of rotation number $\theta$ and we assume that we have an approximation $\theta_{0}$. Then, we suppose that we can select $L \in \mathbb{N}$ (depending on $\mu_{0}$ and $\theta_{0}$ ) in order to unfold the curve and obtain an orbit of a circle map $f=f^{\left(L, \theta_{0}\right)}$ (or circle correspondence), that has the same rotation number $\theta$, given by

$$
x_{n}^{\left(L, \theta_{0}\right)}=\frac{1}{2 \pi} \arctan \frac{\operatorname{Im} z_{n}^{\left(L, \theta_{0}\right)}}{\operatorname{Re} z_{n}^{\left(L, \theta_{0}\right)}}
$$

where $z_{n}^{\left(L, \theta_{0}\right)}$ are given in equation (3). The computation of the derivatives of $x_{n}^{\left(L, \theta_{0}\right)}=f^{n}\left(x_{0}^{\left(L, \theta_{0}\right)}\right)$, that are required to compute $D_{\mu} S_{N}^{p}$, are carried out as

$$
D_{\mu}\left(x_{n}^{\left(L, \theta_{0}\right)}\right)=\frac{1}{2 \pi} \frac{\operatorname{Im}\left(\left(D_{\mu} z_{n}\right)^{\left(L, \theta_{0}\right)}\right) \operatorname{Re} z_{n}^{(L)}-\operatorname{Re}\left(\left(D_{\mu} z_{n}\right)^{\left(L, \theta_{0}\right)}\right) \operatorname{Im} z_{n}^{\left(L, \theta_{0}\right)}}{\left(\operatorname{Re} z_{n}^{\left(L, \theta_{0}\right)}\right)^{2}+\left(\operatorname{Im} z_{n}^{\left(L, \theta_{0}\right)}\right)^{2}},
$$


where

$$
\left(D_{\mu} z_{n}\right)^{\left(L, \theta_{0}\right)}=\frac{1}{L} \sum_{m=n}^{L+n-1} D_{\mu} z_{m} \mathrm{e}^{2 \pi \mathrm{i}(n-m) \theta_{0}} .
$$

Then, given an averaging order $p$, we can compute the sums $D_{\mu} S_{N}^{p}$ that allow to extrapolate $D_{\mu} \theta$ with an error of order $\mathcal{O}\left(1 / N^{p}\right)$. The only point that we need to clarify is the computation of the derivatives $D_{\mu} z_{n}$. They are easily obtained by means of the recursive formula

$$
\operatorname{Re}\left(D_{\mu} z_{n}\right)=\frac{\partial F_{1}}{\partial u}\left(z_{n-1}\right) \operatorname{Re}\left(D_{\mu} z_{n-1}\right)+\frac{\partial F_{1}}{\partial v}\left(z_{n-1}\right) \operatorname{Im}\left(D_{\mu} z_{n-1}\right)
$$

and similarly for $\operatorname{Im}\left(D_{\mu} z_{n}\right)$ replacing $F_{1}$ by $F_{2}$.

Furthermore, if we consider a family of analytic maps $\alpha \in \Lambda \subset \mathbb{R} \mapsto F_{\alpha}$ such that for any $\alpha$ we have a family of invariant curves as described before, i.e., there is a parameter $\mu$ labeling invariant curves of $F_{\alpha}$ in a Cantor set $U_{\alpha}$. This setting induces a function $(\alpha, \mu) \mapsto \theta(\alpha, \mu)$. Omitting the dependence on $(\alpha, \mu)$, let $z_{n}^{\left(L, \theta_{0}\right)}$ be the unfolded iterates of an orbit that belongs to one of the above curves. Then, we can compute the derivative of $\theta$ with respect to $\alpha$ just by averaging the sums of $D_{\alpha}\left(x_{n}^{\left(L, \theta_{0}\right)}\right)$. These iterates are evaluated as explained in the text but using now the recursive formulas

$$
\operatorname{Re}\left(D_{\alpha} z_{n}\right)=\frac{\partial F_{1}}{\partial \alpha}\left(z_{n-1}\right)+\frac{\partial F_{1}}{\partial u}\left(z_{n-1}\right) \operatorname{Re}\left(D_{\alpha} z_{n-1}\right)+\frac{\partial F_{1}}{\partial v}\left(z_{n-1}\right) \operatorname{Im}\left(D_{\alpha} z_{n-1}\right),
$$

and similarly for $\operatorname{Im}\left(D_{\alpha} z_{n}\right)$ replacing $F_{1}$ by $F_{2}$.

The generalization of the previous recurrences to compute high order derivatives of the rotation number is straightforward from Leibniz and product rules (see [23]). We also refer there for details about the use of this information to implement a Newton method for the numerical continuation of invariant curves. In addition, expression (21) allows to obtain (pseudo-analytic) asymptotic expansions relating parameters and initial conditions that correspond to curves of prefixed rotation number (see an application to Hénon's map in [23]).

\subsection{Higher order unfolding of curves}

As it is discused in Section 2.3, if we know the rotation number with an error $\varepsilon$ small enough, then we can select a number $L \in \mathbb{N}$ (depending on $\varepsilon$ ) to unfold the curve obtaining a new curve which is a circle with an error of order $\mathcal{O}(\varepsilon)=\mathcal{O}(1 / L)$ - we refer to the discussion that follows Proposition 2.8. Roughly speaking, in the same way that the method of [29] accelerates the convergence of the definition in (16) to the rotation number from $\mathcal{O}(1 / N)$ to $\mathcal{O}\left(1 / N^{p+1}\right)$, we introduce higher order averages to the iterates $z_{n}^{\left(L, \theta_{0}\right)}$ to accelerate the convergence of the new curve to a circle. Concretely, by performing averages of order $p$ we improve the rate of convergence from $\mathcal{O}(1 / L)$ to $\mathcal{O}\left(1 / L^{p}\right)$. 
Given $\theta_{0} \in \mathbb{R}$, a complex sequence $\left\{z_{n}\right\}_{n \in \mathbb{Z}}$ and a natural number $L$ we introduce the following recursive sums of order $p$

$$
\mathcal{S}_{L, \theta_{0}, n}^{1}=\sum_{m=n}^{L+n-1} z_{m} \mathrm{e}^{-2 \pi \mathrm{i} m \theta_{0}}, \quad \mathcal{S}_{L, \theta_{0}, n}^{p}=\sum_{l=1}^{L} \mathcal{S}_{l, \theta_{0}, n}^{p-1}
$$

and the corresponding averaged sums

$$
\widetilde{\mathcal{S}}_{L, \theta_{0}, n}^{p}=\left(\begin{array}{c}
L+p-1 \\
p
\end{array}\right)^{-1} \mathcal{S}_{L, \theta_{0}, n}^{p} .
$$

Definition 2.17. Under the above conditions, given $p \in \mathbb{N}$, we define the following iterates for any integer $q \geq p$

$$
z_{n}^{\left(2^{q}, \theta_{0}, p\right)}=\left(\sum_{j=0}^{p-1} c_{j}^{(p-1)} \widetilde{\mathcal{S}}_{L_{j}, \theta_{0}, n}^{p}\right) \mathrm{e}^{2 \pi \mathrm{i} n \theta_{0}},
$$

where $L_{j}=2^{q-p+j+1}$ and the coefficients $c_{j}^{(p-1)}$ are given in formula (19).

We remark that $z_{n}^{\left(2^{q}, \theta, 1\right)}=z_{n}^{\left(2^{q}, \theta\right)}$, but that the iterates $z_{n}^{\left(L, \theta_{0}, p\right)}$ are only defined for $L$ being a power of 2, since they are constructed following the ideas in Algorithms 2.14 and 2.16. We see next that if certain non-resonance conditions are fulfilled, these new iterates belong to a quasi-periodic signal such that the corresponding curve approaches a circle improving Proposition 2.8. For the sake of simplicity, we assume non-resonance conditions as those discused in Remark 2.9.

Proposition 2.18. Let $\left\{z_{n}\right\}_{n \in \mathbb{Z}}$ be a quasi-periodic signal of rotation number $\theta \in \mathcal{D}$ and averaging order $p$. Let us consider that $\varepsilon=\theta_{0}-\theta$ is small and that $\theta_{0}$ satisfies

$$
\left|1-\mathrm{e}^{2 \pi \mathrm{i}\left(k \theta-\theta_{0}\right)}\right| \geq \frac{2}{C|k-1|^{\tau}} \quad \forall k \in \mathbb{Z} \backslash\{1\}
$$

for some $C, \tau>0$. Then, there exists a periodic analytic function $\gamma^{\left(2^{q}, \theta_{0}, p\right)}: \mathbb{T} \rightarrow \mathbb{C}$ such that $z_{n}^{\left(2^{q}, \theta_{0}, p\right)}=\gamma^{\left(2^{q}, \theta_{0}, p\right)}(n \theta)$ and it turns out that

$$
\left\|\gamma^{\left(2^{q}, \theta_{0}, p\right)}-\gamma_{1}\left[\hat{\gamma}_{1}^{\left(2^{q}, \theta_{0}, p\right)}\right]\right\|=\mathcal{O}\left(2^{-q p}\right)
$$

where the function $\gamma_{1}[\cdot]$ was introduced in Definition 2.5. Moreover, $\hat{\gamma}_{1}^{\left(2^{q}, \theta_{0}, p\right)}$ - the first Fourier coefficient of $\gamma^{\left(2^{q}, \theta_{0}, p\right)}$ — has the following expression

$$
\hat{\gamma}_{1}^{\left(2^{q}, \theta_{0}, p\right)}=\sum_{j=0}^{p-1} c_{j}^{(p-1)} \widetilde{\Delta}_{L_{j}, \varepsilon}^{p}, \quad L_{j}=2^{q-p+j+1}
$$


where $\widetilde{\Delta}_{L, \varepsilon}^{p}$ is defined recursively as follows

$$
\Delta_{L, \varepsilon}^{1}=\hat{\gamma}_{1} \frac{1-\mathrm{e}^{-2 \pi \mathrm{i} \varepsilon L}}{1-\mathrm{e}^{-2 \pi \mathrm{i} \varepsilon}}, \quad \Delta_{L, \varepsilon}^{p}=\sum_{l=1}^{L} \Delta_{l, \varepsilon}^{p-1}, \quad \widetilde{\Delta}_{L, \varepsilon}^{p}=\left(\begin{array}{c}
L+p-1 \\
p
\end{array}\right)^{-1} \Delta_{L, \varepsilon}^{p} .
$$

In particular, we have that $\lim _{\varepsilon \rightarrow 0} \hat{\gamma}_{1}^{\left(2^{q}, \theta_{0}, p\right)}=\hat{\gamma}_{1}$.

Proof. This result is obtained by means of the same arguments used in [29]. First, we claim that the following expression follows by induction

$$
\widetilde{\mathcal{S}}_{L, \theta_{0}, n}^{p}=\widetilde{\Delta}_{L, \varepsilon}^{p} \mathrm{e}^{-2 \pi \mathrm{i} n \varepsilon}+\left(\sum_{l=1}^{p-1} \frac{\mathcal{A}_{l, \theta_{0}}^{p}(n \theta)}{(L+p-l) \cdots(L+p-1)}+\mathcal{E}_{L, \theta_{0}}^{p}(n \theta)\right) \mathrm{e}^{-2 \pi \mathrm{i} n \theta_{0}},
$$

where the coefficients $\left\{\mathcal{A}_{l, \theta_{0}}^{p}(n \theta)\right\}_{l=1, \ldots, p-1}$ are given by

$$
\mathcal{A}_{l, \theta_{0}}^{p}(n \theta)=(-1)^{l+1}(p-l+1) \cdots p \sum_{k \in \mathbb{Z} \backslash\{1\}} \hat{\gamma}_{k} \frac{\mathrm{e}^{2 \pi \mathrm{i}(l-1)\left(k \theta-\theta_{0}\right)}}{\left(1-\mathrm{e}^{2 \pi \mathrm{i}\left(k \theta-\theta_{0}\right)}\right)^{l}} \mathrm{e}^{2 \pi \mathrm{i} k n \theta}
$$

and the remainder is

$$
\mathcal{E}_{L, \theta_{0}}^{p}(n \theta)=\frac{(-1)^{p+1} p !}{L \cdots(L+p-1)} \sum_{k \in \mathbb{Z} \backslash\{1\}} \hat{\gamma}_{k} \frac{\mathrm{e}^{2 \pi \mathrm{i}(p-1)\left(k \theta-\theta_{0}\right)}\left(1-\mathrm{e}^{2 \pi \mathrm{i} L\left(k \theta-\theta_{0}\right)}\right)}{\left(1-\mathrm{e}^{2 \pi \mathrm{i}\left(k \theta-\theta_{0}\right)}\right)^{p}} \mathrm{e}^{2 \pi \mathrm{i} k n \theta} .
$$

For example, we consider the sum for $p=2$

$$
\begin{aligned}
\mathcal{S}_{L, \theta_{0}, n}^{2}= & \sum_{l=1}^{L} \sum_{m=n}^{l+m-1} z_{m} \mathrm{e}^{-2 \pi \mathrm{i} m \theta_{0}}=\sum_{l=1}^{L} \sum_{m=n}^{l+m-1} \sum_{k \in \mathbb{Z}} \hat{\gamma}_{k} \mathrm{e}^{2 \pi \mathrm{i} m\left(k \theta-\theta_{0}\right)}=\sum_{l=1}^{L} \sum_{m=n}^{l+m-1} \hat{\gamma}_{1} \mathrm{e}^{-2 \pi \mathrm{i} m \varepsilon} \\
& +\sum_{k \in \mathbb{Z} \backslash\{1\}} \hat{\gamma}_{k} \sum_{l=1}^{L} \sum_{m=n}^{l+m-1} \mathrm{e}^{2 \pi \mathrm{i} m\left(k \theta-\theta_{0}\right)}=\Delta_{L, \varepsilon}^{2} \mathrm{e}^{-2 \pi \mathrm{i} n \varepsilon}+L \sum_{k \in \mathbb{Z} \backslash\{1\}} \hat{\gamma}_{k} \frac{\mathrm{e}^{2 \pi \mathrm{i} n\left(k \theta-\theta_{0}\right)}}{1-\mathrm{e}^{2 \pi \mathrm{i}\left(k \theta-\theta_{0}\right)}} \\
& -\sum_{k \in \mathbb{Z} \backslash\{1\}} \hat{\gamma}_{k} \frac{\mathrm{e}^{2 \pi \mathrm{i}(n+1)\left(k \theta-\theta_{0}\right)}\left(1-\mathrm{e}^{2 \pi \mathrm{i} L\left(k \theta-\theta_{0}\right)}\right)}{\left(1-\mathrm{e}^{2 \pi \mathrm{i}\left(k \theta-\theta_{0}\right)}\right)^{2}} .
\end{aligned}
$$

Dividing this expression by $L(L+1) / 2$, we obtain (27) and we proceed inductively to prove the claim. Hence, it is clear that the sequence $z_{n}^{\left(2^{q}, \theta_{0}, p\right)}$ in (23) corresponds to a quasi-periodic signal since it is a linear combination of quasi-periodic functions.

Using the analyticity assumptions and estimates in (24) it turns out that the obtained remainder is of order $\mathcal{E}_{L, \theta_{0}}^{p}(n \theta)=\mathcal{O}\left(1 / L^{p}\right)$. To extrapolate in this expression using the coefficients (19) we require the denominators $(L+p-l) \cdots(L+p-1)$ in (27) not to depend on $p$. To this end, we write

$$
\widetilde{\mathcal{S}}_{L, \theta_{0}, n}^{p}=\widetilde{\Delta}_{L, \varepsilon}^{p} \mathrm{e}^{-2 \pi \mathrm{i} n \varepsilon}+\left(\sum_{l=1}^{p-1} \frac{\hat{\mathcal{A}}_{l, \theta_{0}}^{p}(n \theta)}{L^{l}}+\hat{\mathcal{E}}_{L, \theta_{0}}^{p}(n \theta)\right) \mathrm{e}^{-2 \pi \mathrm{i} n \theta_{0}}
$$


by redefining the coefficients $\left\{\hat{\mathcal{A}}_{l, \theta_{0}}^{p}(n \theta)\right\}_{l=1, \ldots, p-1}$, also independent of $L$, where $\hat{\mathcal{E}}_{L, \theta_{0}}^{p}(n \theta)$ differs from $\mathcal{E}_{L, \theta_{0}}^{p}(n \theta)$ only by terms of order $\mathcal{O}\left(1 / L^{p}\right)$. Hence, we can use Richardson extrapolation using a maximum number of iterates $L=2^{q}$ and introduce the corresponding expression into (23), thus obtaining

$$
z_{n}^{\left(2^{q}, \theta_{0}, p\right)}=\left(\sum_{j=0}^{p-1} c_{j}^{(p-1)} \widetilde{\Delta}_{L_{j}, \varepsilon}^{p}\right) \mathrm{e}^{2 \pi \mathrm{i} n \theta}+\sum_{j=0}^{p-1} c_{j}^{(p-1)} \hat{\mathcal{E}}_{L_{j}, \theta_{0}}^{p}(n \theta)=\gamma^{\left(2^{q}, \theta_{0}, p\right)}(n \theta) .
$$

Therefore, the estimate (25) is obtained after observing that the first Fourier coefficient is given by equation (26). Finally, the $\operatorname{limit}_{\lim _{\varepsilon \rightarrow 0}} \hat{\gamma}_{1}^{\left(2^{q}, \theta_{0}, p\right)}=\hat{\gamma}_{1}$ follows from the fact that $\sum_{j=0}^{p-1} c_{j}^{(p-1)}=1$.

\subsection{Extrapolation of Fourier coefficients}

Our goal now is to adapt the previous methodology in order to obtain the Fourier coefficients of a quasi-periodic signal of known rotation number. Let us recall that standard FFT algorithms are based in equidistant samples of points. Since the iterates of a quasi-periodic signal are not distributed in such a way on $\mathbb{T}$, one has to implement a non-equidistant FFT or resort to interpolation of points (see for instance $[1,8,25]$ ). We avoid this difficulty using the fact that the iterates are equidistant "according with the quasi-periodic dynamics".

We consider a quasi-periodic signal $z_{n}=\gamma(n \theta)$ of rotation number $\theta \in \mathcal{D}$ as given by Definition 2.1. Let us observe that we can compute the $t$-th Fourier coefficient, $\hat{\gamma}_{t}$, as the average of the quasi-periodic signal $z_{n} \mathrm{e}^{-2 \pi \mathrm{int} t}$. For this purpose, we introduce the following recursive sums of order $p$

$$
\mathfrak{S}_{N, t}^{0}=z_{N} \mathrm{e}^{-2 \pi \mathrm{i} N t \theta}, \quad \mathfrak{S}_{N, t}^{p}=\sum_{n=1}^{N} \mathfrak{S}_{n, t}^{p-1},
$$

and their corresponding averages

$$
\widetilde{\mathfrak{S}}_{N, t}^{p}=\left(\begin{array}{c}
N+p-1 \\
p
\end{array}\right)^{-1} \mathfrak{S}_{N, t}^{p}
$$

Proposition 2.19. For any analytic quasi-periodic signal $z_{n}=\gamma(n \theta)$ of rotation number $\theta \in \mathcal{D}$ the following expression is satisfied

$$
\widetilde{\mathfrak{S}}_{N, t}^{p}=\hat{\gamma}_{t}+\sum_{l=1}^{p-1} \frac{\mathfrak{A}_{t, l}^{p-1}}{N^{l}}+\mathfrak{E}_{t}^{p}(N)
$$

where the coefficients $\mathfrak{A}_{t, l}^{p}$ are independent of $N$. Furthermore, the remainder $\mathfrak{E}_{t}^{p}(N)$ is uniformly bounded by an expression of order $\mathcal{O}\left(1 / N^{p}\right)$. 
This proposition, that can be proved analogously as Proposition 2.13, allows to obtain the following extrapolation scheme to approximate $\hat{\gamma}_{t}$.

Algorithm 2.20. Once an averaging order $p$ is selected, we take $N=2^{q}$ iterates of the map, for some $q>p$, and compute the sums $\left\{\mathfrak{S}_{N_{j}, t}^{p}\right\}_{j=0, \ldots, p}$ with $N_{j}=2^{q-p+j+1}$. We approximate the th Fourier coefficient using the formula

$$
\hat{\gamma}_{t}=\Phi_{q, p, t}+\mathcal{O}\left(2^{-p q}\right), \quad \Phi_{q, p, t}=\sum_{j=0}^{p-1} c_{j}^{(p-1)} \widetilde{\mathfrak{S}}_{N_{j}, t}^{p},
$$

using the same formula (19) for the coefficients $c_{j}^{(p-1)}$.

The extrapolation error of this algorithm can be estimated by means of the following (heuristic) expression

$$
\left|\hat{\gamma}_{t}-\Phi_{q, p, t}\right| \leq \frac{\nu}{2^{p}}\left|\Phi_{q, p, t}-\Phi_{q-1, p, t}\right| .
$$

Remark 2.21. As it was mentioned in the introduction, the typical approach to compute an invariant curve it to look for it in terms of its Fourier representation. One of the main features of the methodology discused in this paper is that we can compute these objects looking for an initial condition on the curve (see Section 3.3 and also examples in [23, 29]) without computing simultaneously any Fourier expansion or similar approximation. Then, the method discussed above allows to obtain "a-posteriori" Fourier coefficients of the parameterization from the iterates of the mentioned initial condition.

Remark 2.22. If we want to compute $M$ Fourier coefficients, notice that Algorithm 2.20 involves a computational cost of order $\mathcal{O}(N M)$ that seems to be deceiving when comparing with FFT methods. Nevertheses, it is clear that the sums (28) can be also computed as it is standard in FFT since they also satisfy Danielson-Lanczos Lemma (see for example [28]), thus obtaining a cost of order $\mathcal{O}(N \log N)$ for computing $N$ coefficients. However, unlike in the other algorithms presented in this paper, this fast implementation requires to store the iterates of the map.

Remark 2.23. We point out that with Algorithm 2.20 we can compute isolated coefficients, meanwhile FFT computes simultaneously all the coefficients up to a given order. This can be useful if one is interested only in computing families of coefficients with high precision, as for example it is done in [30] —using similar ideas_for coefficients corresponding to Fibonacci numbers.

\section{Some numerical illustrations}

In this part of the paper we illustrate several features of the methods discused in Section 2. To this end, we have selected three different contexts that we summarize next. 
- First, in Section 3.1, we study invariant curves inside the Siegel domain of a quadratic polynomial. We use this example, where the rotation number is known "a-priori", as a test of the methods. In particular, we show how difficult it is to unfold a given invariant curve as a function of the arithmetic properties of the selected rotation numbers. Furthermore, we introduce two simple criteria to decide if the projection of the iterates of the invariant curve induces a circle map.

- Then, in Section 3.2, we deal with a toy model obtained by fixing the Fourier coefficients and the rotation number that define a non-embedded quasi-periodic signal. In this example we study the behavior of the unfolded curve $\gamma^{\left(L, \theta_{0}\right)}$ and we check the estimates in Lemma 2.6 and Proposition 2.8. Furthermore, in order to simulate uncertainty coming from experimental data we add to this signal a normally distributed random error and we show that the method still provides very accurate results to compute the rotation number of the signal.

- In Section 3.3 we consider the study of quasi-periodic invariant curves for planar nontwist maps. For the standard non-twist map, we apply Algorithm 2.14 to compute the rotation number in cases where we can compute easily the "lift" of the circle correspondence induced by the direct projection, and also in a very folded curve that we require to unfold. Moreover, we unfold a shearless invariant curve comparing the methods in Sections 2.3 and 2.6. For Hénon's map, we apply Algorithm 2.16 to illustrate the computations of derivatives of the rotation number from the "lift" of circle correspondences induced by a family of invariant curves. Finally, we use our methodology to continue numerically a folded (labyrinthic) invariant curve in a more degenerate family of maps.

Let us observe that, since all the recursive sums are evaluated using lifts rather than maps, they turn out to be very large when we increase the order of averaging and the number of iterates. Concretely,

$$
S_{N}^{p}=\mathcal{O}\left(N^{p+1}\right), \quad D_{\mu}^{d} S_{N}^{p}=\mathcal{O}\left(N^{p+1}\right), \quad \mathcal{S}_{L, \theta_{0}, n}^{p}=\mathcal{O}\left(L^{p}\right), \quad \mathfrak{S}_{N}^{p}=\mathcal{O}\left(N^{p}\right)
$$

A natural way to overcome this problem is to do computations by using a representation of real numbers using a computer arithmetic having a large number of decimal digits. Moreover, we have to be very careful with the manipulation of this large numbers to prevent the loss of significant digits (for example, by storing separately integer and decimal parts) and beware not to "saturate" them.

The presented computations have been performed using a $\mathrm{C}++$ compiler and multiple arithmetic (when it is required) has been provided by the routines double-double and quad-double package of [18], which include a double-double data type of approximately 32 decimal digits and a quadruple-double data type of approximately 64 digits. 

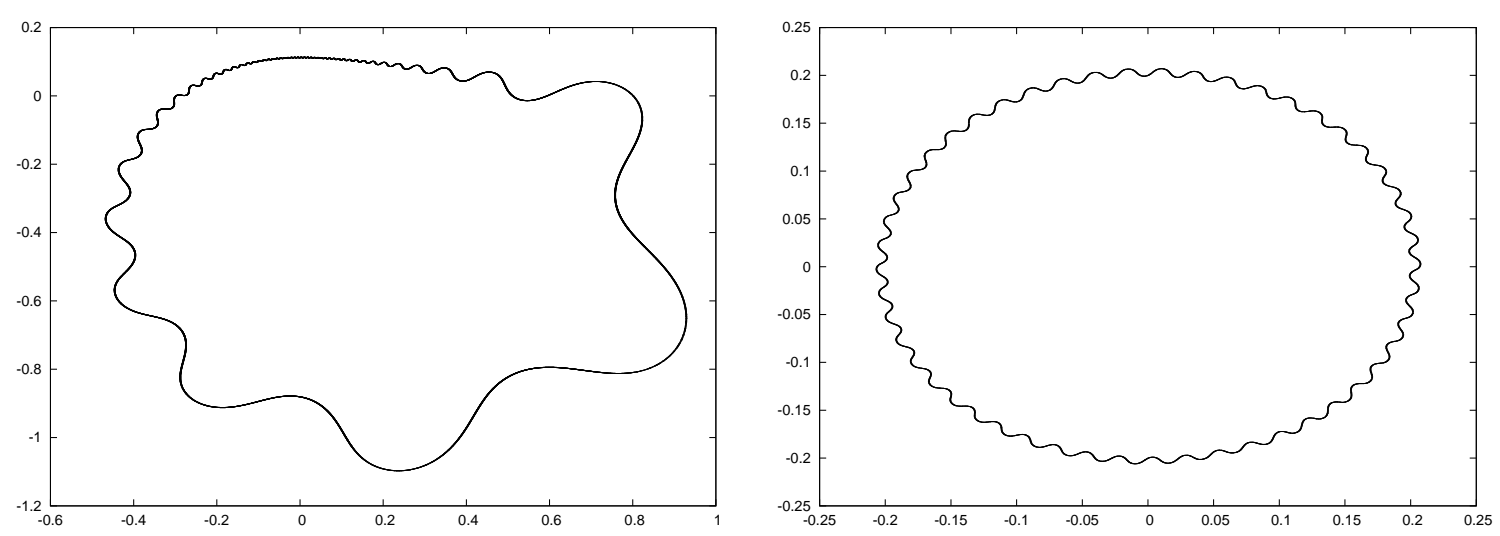

Figure 3: Left: iterates (in the complex plane) of the point $z_{0}=0.8$ for the quadratic polynomial with $\theta=\theta^{(50)}$. Right: averaged iterates $z_{n}^{\left(200, \theta^{(50)}\right)}$ of this curve given by (3).
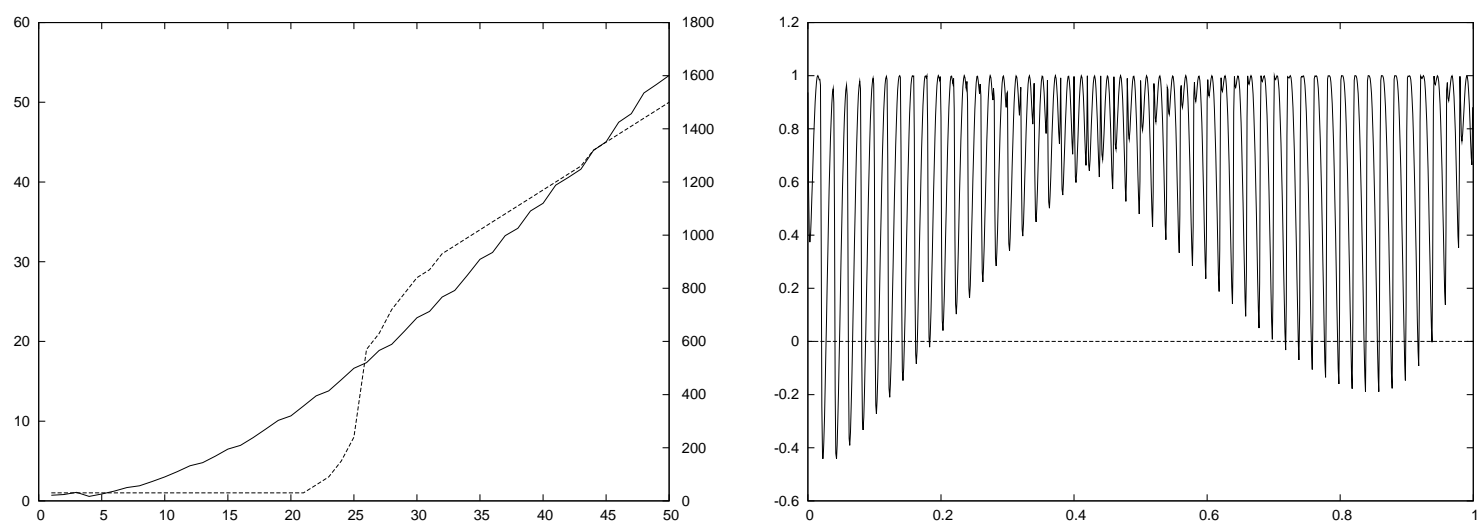

Figure 4: Unfolding of the invariant curve corresponding to the point $z_{0}=0.8$ for the quadratic polynomial. Left plot: we show, versus the integer $s$, the value of $L$ for which the curve $\gamma^{\left(L, \theta^{(s)}\right)}$ is "almost" a circle (solid line, right vertical axis) and the minimum value of $L$ for which the projection defines a circle map (dashed line, left vertical axis). Right plot: we plot function (30) for the averaged curve of Figure 3 (left plot) versus the arc parameter $\alpha$ on $\mathbb{T}$ described in Remark 3.1.

\subsection{Siegel domain of a quadratic polynomial}

Let $F: U \rightarrow \mathbb{C}$ be an analytic map, where $U \subset \mathbb{C}$ is an open set, such that $F(0)=0$ and $F^{\prime}(0)=\mathrm{e}^{2 \pi \mathrm{i} \theta}$. It is well known that if $\theta$ is a Brjuno number, then there exists a conformal isomorphism that conjugates $F$ to a rotation around the origin of angle $2 \pi \theta$ (see [38]). The conjugation determines a maximal set (called Siegel disk) which is foliated by invariant curves of rotation number $\theta$.

In particular, we consider the case of the quadratic polynomial $F(z)=\lambda\left(z-\frac{1}{2} z^{2}\right)$, with $\lambda=\mathrm{e}^{2 \pi \mathrm{i} \theta}$, for several rotation numbers $\theta$. Concretely, we use $\theta=\theta^{(s)} \in(0,1)$ which is a zero of $\theta^{2}+s \theta-1=0$, with $s \in \mathbb{N}$. It is clear that $\theta^{(s)}$ is a Diophantine number for any $s$ but with a 
larger constant $C$ (recall Definition 2.3) when $s$ increases. Note that $\theta^{(s)}=1 / s-6 / s^{3}+\mathcal{O}\left(1 / s^{5}\right)$ shows that, for large $s, \theta^{(s)}$ is "close" to a rational number.

Even in this simple example, the direct projection on the angular variable does not always give a diffeomorphism on $\mathbb{T}$. For example, in the left plot of Figure 3 we show the curve that corresponds to $\theta=\theta^{(50)}$ for the initial condition $z_{0}=0.8$. The right plot of Figure 3 corresponds to the averaged iterates $z_{n}^{\left(200, \theta^{(50)}\right)}$ according to (3). As expected, the new curve is closer to a circle centered at the origin.

Our first goal is to emphasize how difficult it becomes to unfold the invariant curve of the point $z_{0}=0.8$ depending on the chosen rotation number $\theta^{(s)}$ (as $s$ increases). To this end, we introduce a criterion to decide when the curve is "close enough" to be a circle. Given a fixed value of $\theta^{(s)}$, we choose $L=1$ and compute $\left\{z_{n}^{\left(L, \theta^{(s)}\right)}\right\}_{n=1, \ldots, 50000}$ iterates, the mean value of their modulus, and the corresponding standard deviation. Then, if the relative standard deviation is less than $0.5 \%$, we consider that the curve $\gamma^{\left(L, \theta^{(s)}\right)}$ is close enough to be a circle or we increase $L$ otherwise. The continuous line in the left plot of Figure 4 (using the vertical axis on the right) shows the obtained value of $L$ versus the integer $s$ that labels the rotation number $\theta^{(s)}$. As expected, we require larger values of $L$ when the rotation number is closer to a rational number.

Let us observe that in practice we do not require to take such large values of $L$ to unfold the curve. Rather than obtaining a circle, we are interested in unfolding the curve in a way that can be projected smoothly into a circle. To this end, we propose a simple criterion to decide if the curve is already unfolded or not. This can be done by computing the changes of sign of the function

$$
z \in \gamma(\mathbb{T}) \longmapsto \operatorname{det}\left(v_{t}(z), v_{r}(z)\right) \in[-1,1],
$$

where $v_{t}(z)$ is the oriented (in the sense of the dynamics) unitary tangent vector of $\gamma$ at the point $z$ and $v_{r}(z)$ the corresponding unitary radial vector with respect to the origin. It is clear that if $\operatorname{det}\left(v_{t}(z), v_{r}(z)\right)$ changes sign at some point, then the curve $\gamma$ is still folded. Moreover, if $\gamma(\mathbb{T})$ is exactly a circle, we have that $\operatorname{det}\left(v_{t}(z), v_{r}(z)\right)$ is constant for all $z \in \gamma(\mathbb{T})$, taking the value -1 or 1 , depending if the iterates rotate clockwise or counterclockwise.

As an example, we apply this criterion to the invariant curve shown in the left plot of Figure 3. The function (30) is shown in the right plot of Figure 4. The horizontal axis in this plot corresponds to the sampling of points on the curve distributed according to Remark 3.1. Let us observe that this function oscillates due to the folds of the invariant curve, and there are changes of sign since the projection is not well posed. Now, we unfold this invariant curve for different values of $\theta^{(s)}$ looking for the minimum value of $L$ such that $\min \left(\operatorname{det}\left(v_{t}(z), v_{r}(z)\right)\right)>0$. The discontinuous line in the left plot of Figure 4 (using the vertical axis on the left) shows this value of $L$ versus $s$, and we observe that it is much smaller than the value of $L$ for which the curve is almost a circle.

Remark 3.1. Function (30) is evaluated by computing the tangent vector $v_{t}(z)$ using finite differences. To this end, we require a good distribution of points along the curve $\gamma$ that are obtained using the fact that we know the rotation number (at least approximately). In particular, 


\begin{tabular}{|cccc|}
\hline coefficient & value & estimated error & real error \\
\hline$\hat{\gamma}_{-1}$ & $1.4-2 \mathrm{i}$ & $6 \cdot 10^{-40}$ & $1 \cdot 10^{-40}$ \\
$\hat{\gamma}_{0}$ & $4.1+1.34 \mathrm{i}$ & $6 \cdot 10^{-41}$ & $9 \cdot 10^{-42}$ \\
$\hat{\gamma}_{1}$ & $-2+2.412 \mathrm{i}$ & $5 \cdot 10^{-41}$ & $8 \cdot 10^{-42}$ \\
$\hat{\gamma}_{2}$ & $-2.5-1.752 \mathrm{i}$ & $4 \cdot 10^{-40}$ & $8 \cdot 10^{-41}$ \\
\hline
\end{tabular}

Table 1: Fourier coefficients defining expression (31) and the numerical error obtained in their approximation using the method of Section 2.7. The estimated error is obtained by means of formula (29).

we fix a number of points $M$ to discretize the curve parameterized by an "arc parameter" $\alpha \in[0,1)$ on $\mathbb{T}$ defined by the quasi-periodic dynamics. We start with a point $z_{0} \in \gamma(\mathbb{T})$ that we identify with the reference parameter $\alpha=0$. Then, we compute the next iterate of the map and we update the parameter $\alpha \leftarrow \alpha+\theta_{0}(\bmod 1)$. Defining $i$ as the integer part of $\alpha M$ and if $d_{\mathbb{T}}(\alpha, i / M)<10^{-4}$ we store the iterate in the position $i$-th of an array. We iterate this process till we store $M$ points on $\gamma(\mathbb{T})$. Observe that these computed points are ordered following the dynamics of the curve so we can compute the tangent vector just by finite differences. We will consider that $\min \left(\operatorname{det}\left(v_{t}(z), v_{r}(z)\right)\right)>0$ if the minimum value at the $M$ selected points is positive.

\subsection{Study of a quasi-periodic signal}

We consider a quasi-periodic signal $z_{n}=\gamma(n \theta)$, with $\theta=(\sqrt{5}-1) / 2 \in \mathcal{D}$, as introduced in Definition 2.1. Interest is focused in the case where $\gamma$ is not an embedding, and hence the corresponding orbit is not related to a planar map. In particular, we consider

$$
\gamma(x)=\hat{\gamma}_{-1} \mathrm{e}^{-2 \pi \mathrm{i} x}+\hat{\gamma}_{0}+\hat{\gamma}_{1} \mathrm{e}^{2 \pi \mathrm{i} x}+\hat{\gamma}_{2} \mathrm{e}^{4 \pi \mathrm{i} x},
$$

where the Fourier coefficients, given in Table 1, have been selected in such a way that the curve $\gamma(\mathbb{T})$ intersects itself.

First, we show the initial curve $\gamma(\mathbb{T})$ (left plot of Figure 5) and averaged curves $\gamma^{(L, \theta)}(\mathbb{T})$ (right plot of Figure 5) corresponding to $L=2,3,4$. Observe that we are using the exact value of the rotation number to compute the averaged iterates given by (3). As expected, the new curves are unfolded and become close to a circle.

Since in this problem we know the rotation number, we can perform a simple test of the method presented in Section 2.7 computing the non-zero Fourier coefficients of the initial curve. To this end we use 64-digit arithmetics (quadruple-double data type from [18]) taking an averaging order $p=9$ and $N=2^{23}$ iterates of the map. Both the estimated extrapolation error using (29) and the real one are shown in Table 1 observing a very good agreement.

Our goal now is to study the dependence with respect to $L$ of the norms in Lemma 2.6 and Proposition 2.8. In Figure 6 (left) we plot the function

$$
L \longmapsto \log _{10}\left(\frac{\left\|\gamma^{(L, \theta)}-\gamma_{1}\left[\hat{\gamma}_{1}\right]\right\|}{\left|\hat{\gamma}_{1}\right|}\right),
$$



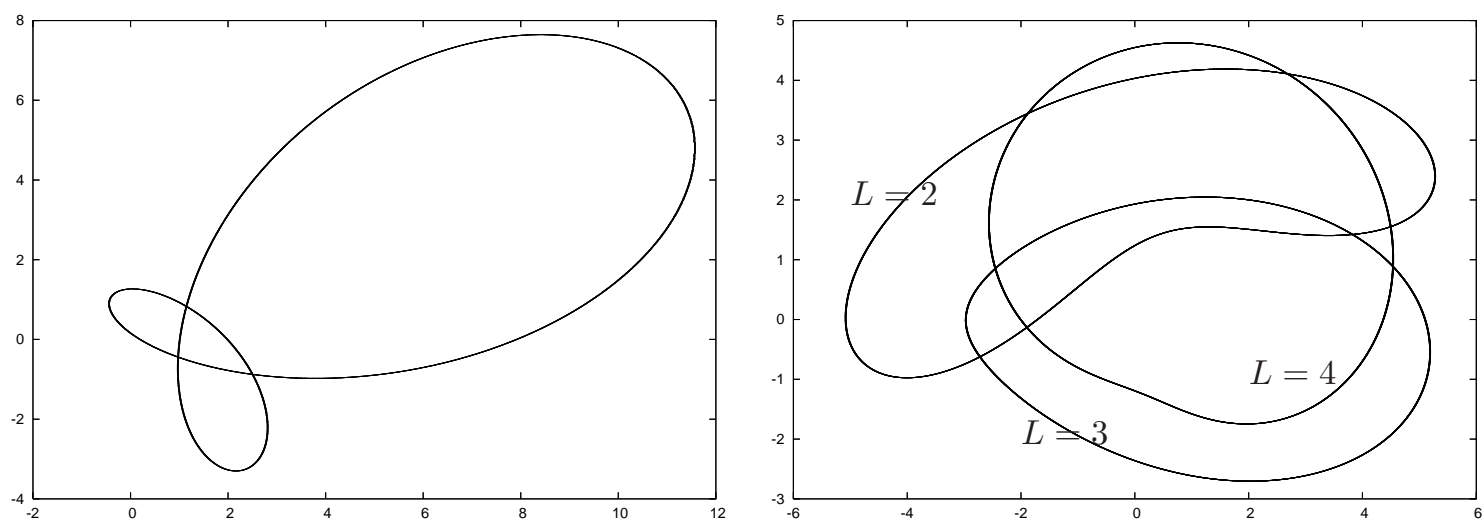

Figure 5: Left: Curve $\gamma(\mathbb{T})$ in the complex plane corresponding to the parameterization in (31). Right: Unfolded curves $\gamma^{(L, \theta)}(\mathbb{T})$, for $L=2,3$ and 4 , using the known value of the rotation number $\theta=(\sqrt{5}-1) / 2$.
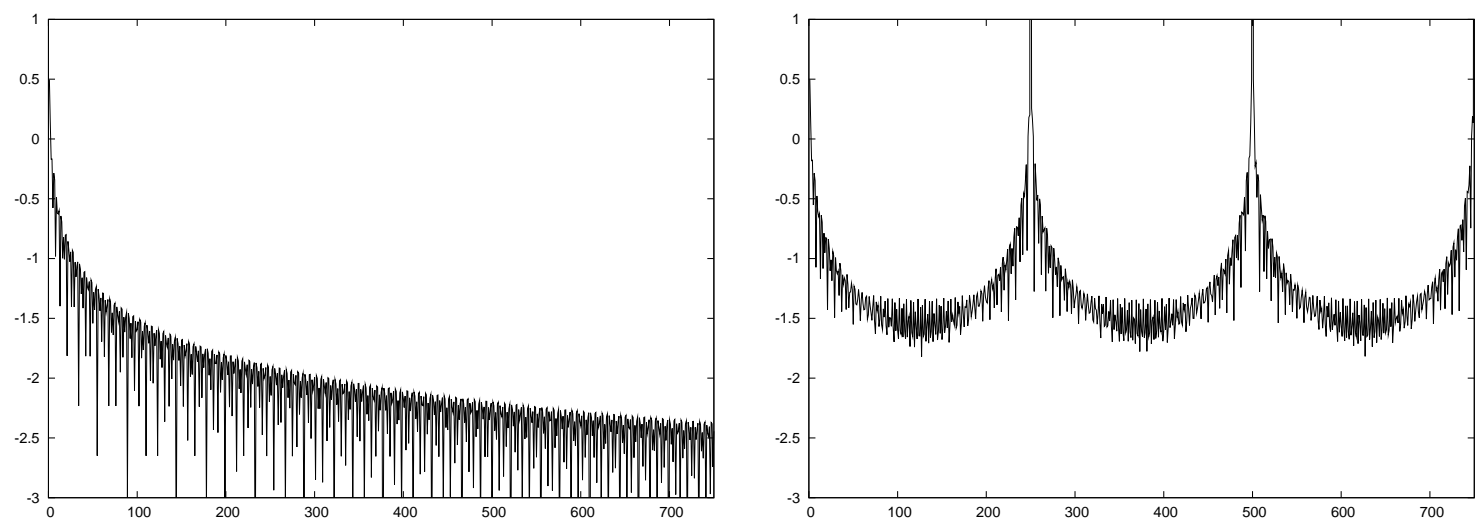

Figure 6: Left: we plot function (32) versus $L$. Right: we plot function (33) versus $L$ using the approximation $\theta_{0}=\theta+1 / 250$.

that can be evaluated from expression (5) using the exact value of the rotation number. We observe that the computed points can be bounded from above in a sharp way by $3.19 / \mathrm{L}$.

On the other hand, assuming that we only have an approximation $\theta_{0}$ of the rotation number $\theta$, we want to study the estimate (8) of Proposition 2.8. Concretely, we compute the function

$$
L \longmapsto \log _{10}\left(\frac{\left\|\gamma^{\left(L, \theta_{0}\right)}-\gamma_{1}\left[\hat{\gamma}_{1}^{\left(L, \theta_{0}\right)}\right]\right\|}{\left|\hat{\gamma}_{1}^{\left(L, \theta_{0}\right)}\right|}\right)
$$

using the approximation $\theta_{0}=\theta+1 / 250$. In the right plot of Figure 6 we observe that this function is close to be periodic, of period approximately 250 , and it reaches a minimum at $L \approx 125(\bmod 250)$ so the bound given in Proposition 2.8 turns out to be quite good in this case.

It is very interesting to study the effect of a random error in the evaluation of iterates, trying 

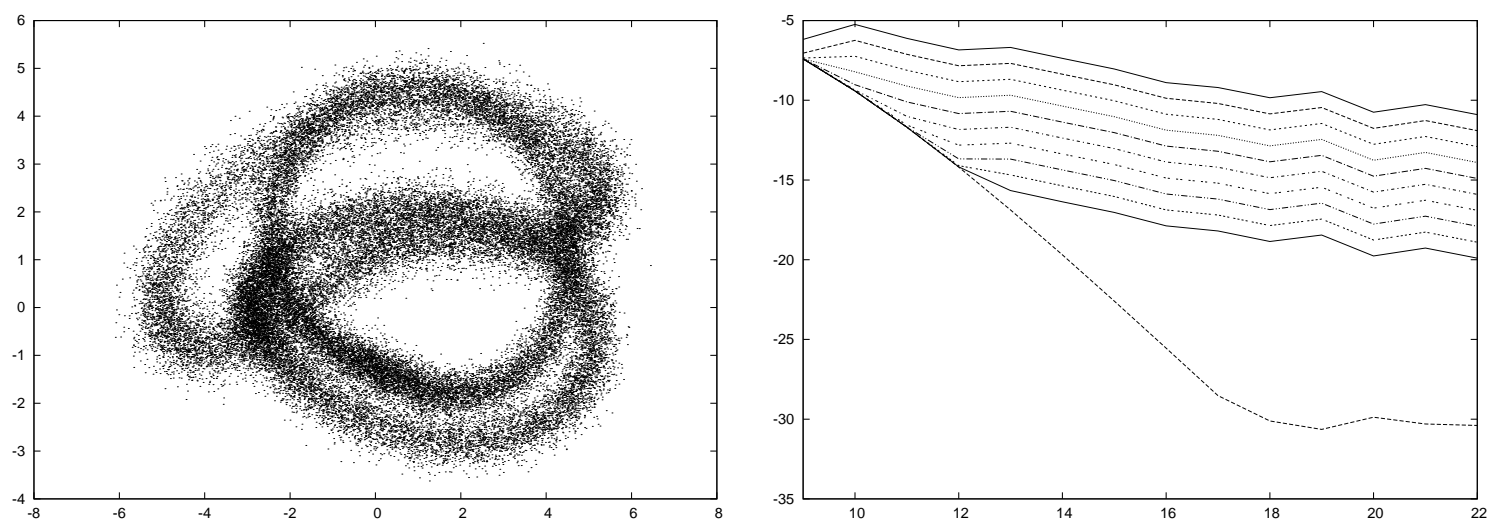

Figure 7: Effect of a random noise in the quasi-periodic signal (31). Left: Unfolded clouds of points (in the complex plane) corresponding to the curves in the right plot of Figure 5 using $\varepsilon=0.5$ (see text for details). Right: For different noises, taken as $\varepsilon=10^{-\delta}$ for $\delta=1, \ldots, 10, \infty$, we plot $\log _{10}$ of the real error versus $q$ in the computation of the rotation number (using $p=9$ and $2^{q}$ iterates). The data is "unfolded" using $L=10$ and $\theta_{0}=\theta+1 / 250$.

to simulate that the source of our quasi-periodic signal is experimental data. Concretely, we consider the iterates $z_{n}=\gamma(n \theta)+\varepsilon x_{n}$, where the real and the imaginary parts of the noise $x_{n}$ are normally distributed with zero mean and unit variance. Of course, the new iterates $z_{n}$ do not belong to a curve but they are distributed in a cloud around the curve in Figure 5 (left plot). If we compute the iterates $z_{n}^{\left(L, \theta_{0}\right)}$, using the approximation $\theta_{0}=\theta+1 / 250$, then it turns out that we can "unfold" the cloud of points in a similar way. For example, in the left plot of Figure 7 we show unfolded clouds for an error of size $\varepsilon=0.5$, using $L=2,3$, 4 , i.e., the same values that we used in Figure 5 (right plot).

Now we focus on the effect of this external noise when computing the rotation number $\theta$ of the "circle map" thus obtained. The size of the considered noise ranges as $\varepsilon=10^{-\delta}$ for $\delta=1, \ldots, 10, \infty$. Although we observe in Figures 5 (right plot) and 7 (left plot) that the "projection" is well defined for $L=4$, in the following computations we take $L=10$ since for this value the corresponding curve is almost a circle. To the constructed "circle map" we apply Algorithm 2.14 to refine the numerical computation of the rotation number. As implementation parameters we take an averaging order $p=9$ and $N=2^{q}$ iterates of the map, with $q=$ $9, \ldots, 22$. The random numbers $x_{n}$ are generated using the routine gasdev from [28] for generating normal (Gaussian) deviates. Computations are performed using 32-digit arithmetics (double-double data type from [18]).

In the right plot of Figure 7 we show, in $\log _{10}$ scale, the error in the computation of the rotation number with respect to $q$ for different values of $\varepsilon$. Let us observe that for $\varepsilon=0$ (lowest curve) the extrapolation error is saturated around $10^{-31}$ for $q \geq 18$. We notice that this error is of the order of the selected arithmetics. The other curves in the right Figure 7 correspond to increasing values of $\varepsilon$ (from bottom to top). Let us remark that in all cases the random error is 

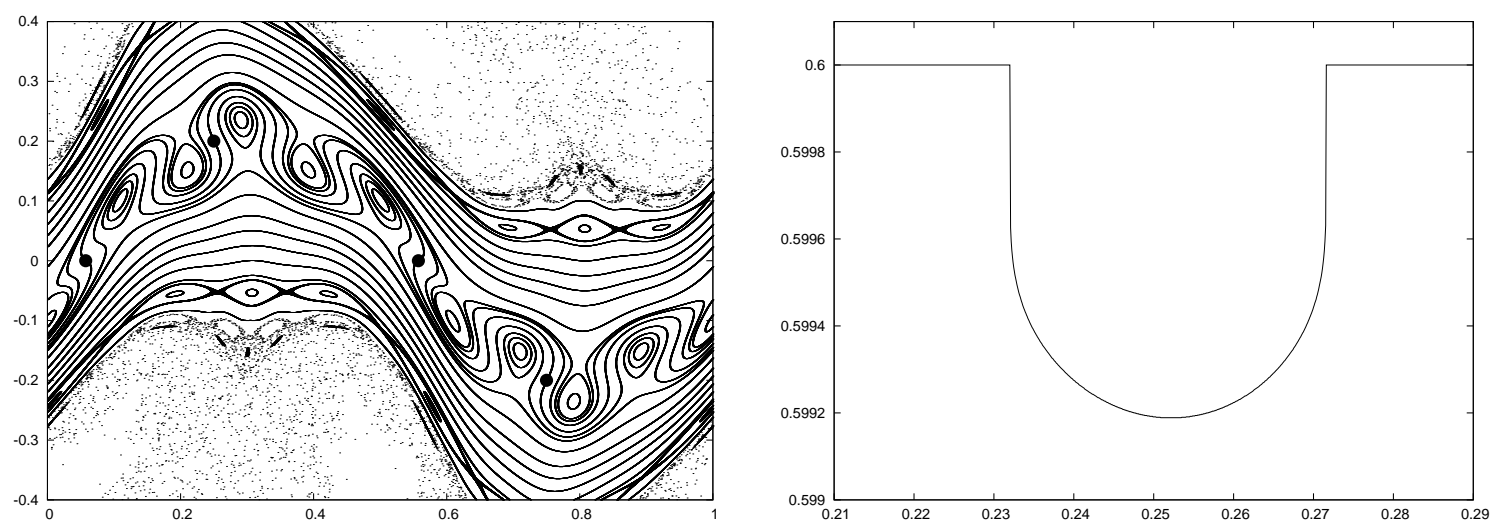

Figure 8: Left: some meandering curves, in the $x y$-plane, of the map (34) around the shearles invariant curve corresponding to $a=0.615$ and $b=0.4$; four dots represent the corresponding indicator points for this curve. Right: Rotation number versus $x$ along the straight line connecting the points $(x, y)=(0.21,0.15)$ and $(x, y)=$ $(0.29,0.235)$ in the left plot.

averaged in a very efficient way, and it turns out that the rotation number is approximated with an error of order $\varepsilon \cdot 10^{-10}$.

\subsection{Study of invariant curves in non-twist maps}

Finally, we apply the developed methodology to the study of quasi-periodic invariant curves of non-twist maps. It is known that Aubry-Mather variational theory for twist maps does not generalize to the non-twist case, but there is an analogue of KAM theory (see for example the works of $[11,34])$. However, the loss of the twist condition introduces different properties than in the twist case, for example the fact that the Birkhoff Graph Theorem does not generalize. A classical mechanism that creates folded invariant curves is called reconnection. Reconnection is a global bifurcation of the invariant manifolds of two or more distinct hyperbolic periodic orbits having the same winding number (we refer to [10, 34, 37] and references therein for discussion of this bifurcation).

Let us start by considering the family of area preserving non-twist maps given by

$$
F_{a, b}:(x, y) \longmapsto(\bar{x}, \bar{y})=\left(x+a\left(1-\bar{y}^{2}\right), y-b \sin (2 \pi x)\right),
$$

where $(x, y) \in \mathbb{T} \times \mathbb{R}$ are phase space coordinates and $a, b$ parameters. This family is usually called standard non-twist map and it is studied as a paradigmatic example of a non-twist family. Although this family is non-generic (it is degenerate in the sense that it contains just one harmonic), it describes the essential features of non-twist systems with a local quadratic extremum in the rotation number.

It is clear that the standard non-twist map violates the twist condition along the curve $y=$ $b \sin (2 \pi x)$ which is called non-monotone curve. Only orbits with points falling on this curve and 
orbits with points on both sides of it are affected by the non-twist property. Among these orbits, of special interest is the one that corresponds to an invariant curve having a local extremum in the rotation number, called shearless invariant curve $\gamma_{S}$. For the standard non-twist map this curve is characterized by the fact that, when it exists, it must contain the points

$$
\left(x_{ \pm}^{(0)}, y_{ \pm}^{(0)}\right)=\left( \pm \frac{1}{4}, \pm \frac{b}{2}\right) \quad\left(x_{ \pm}^{(1)}, y_{ \pm}^{(1)}\right)=\left(\frac{a}{2} \pm \frac{1}{4}, 0\right)
$$

that are called indicator points (see [31]). These points are used extensively in the literature to study the breakdown of shearless invariant curves (we refer for example to $[1,37]$ ).

In the left plot of Figure 8 we show some invariant curves close to a reconnection scenario for $a=0.615$ and $b=0.4$. We observe some meandering curves, i.e., curves that are folded around periodic orbits in such a way that they are not graphs over $x$. In addition, we plot the four indicator points in order to identify the shearless invariant curve. Actually, the invariant curve that we used as an illustration in Section 2.1 (see Figure 1) is precisely this shearless curve in the complex variable $z=\mathrm{e}^{y} \mathrm{e}^{\mathrm{i} x}$.

First, we focus on this shearless curve computing its rotation number by applying the extrapolation method of Algorithm 2.14 to the circle correspondence obtained by direct projection (see the discussion in Sections 1 and 2.1) on the angular variable $x$. Since the folds of this example are relatively small and the rotation number is quite big - it is close to 0.6 , i.e., the winding number of the nearby periodic orbits - this direct projection allows to compute numerically the lift of this circle correspondence without unfolding the curve. In Table 2 we give the estimated extrapolation error, by means of formula (20), in the computation of the rotation number of $\gamma_{S}$, for different values of the extrapolation order $p$ and number of iterates $2^{q}$. Computations are performed using 32-digit arithmetics (double-double data type from [18]). Let us observe that the extrapolation method allows to obtain a very good approximation of the rotation number with a relative small number of iterates, in contrast with $p=0$ which corresponds to the definition of the rotation number - let us mention that some recent works like [37] use the definition to approximate the rotation number. According to our estimates, the best computed approximation of the rotation number turns out to be

$$
\theta \simeq \Theta_{21,7}=0.59918902772269558576430971159247
$$

In addition, we compute the rotation number of meandering curves in the left plot of Figure 8 using an averaging order $p=7$ and $2^{21}$ iterates of the map. In Figure 8 (right plot) we show the rotation number profile in this reconnection scenario. Concretely, we compute the rotation number for the orbits corresponding to 1000 points along an straight line connecting $(x, y)=$ $(0.21,0.15)$ and $(x, y)=(0.29,0.235)$, that are close to the elliptic periodic orbits of winding number $3 / 5$. As far as the estimated extrapolation error is concerned, $93 \%$ of the points have an error less than $10^{-26}$ and $98 \%$ of the points have an error less than $10^{-20}$. The minimum value in the profile corresponds to the rotation number of $\gamma_{S}$, and we observe the loss of uniqueness of invariant curves when the twist condition fails. 


\begin{tabular}{|c|c|c|c|c|c|c|c|c|c|c|c|c|}
\hline$p$ & $q=10$ & $q=11$ & $q=12$ & $q=13$ & $q=14$ & $q=15$ & $q=16$ & $q=17$ & $q=18$ & $q=19$ & $q=20$ & $q=21$ \\
\hline 0 & $1.4 \mathrm{e}-03$ & $7.3 e-04$ & $1.9 \mathrm{e}-04$ & $1.9 \mathrm{e}-04$ & $8.1 \mathrm{e}-05$ & $3.6 \mathrm{e}-05$ & $1.1 \mathrm{e}-05$ & $1.0 \mathrm{e}-05$ & $5.0 \mathrm{e}-06$ & $2.5 e-06$ & $1.0 \mathrm{e}-06$ & $3.4 \mathrm{e}-07$ \\
\hline 1 & $7.9 \mathrm{e}-06$ & $5.5 e-06$ & $2.6 \mathrm{e}-07$ & $8.1 \mathrm{e}-07$ & $1.4 \mathrm{e}-07$ & $6.9 \mathrm{e}-08$ & $3.7 e-09$ & $1.9 \mathrm{e}-09$ & $1.2 \mathrm{e}-09$ & $2.5 e-11$ & $8.8 \mathrm{e}-12$ & $6.4 \mathrm{e}-12$ \\
\hline 2 & $3.8 \mathrm{e}-07$ & $8.6 e-09$ & $3.0 \mathrm{e}-08$ & $4.5 \mathrm{e}-08$ & $4.6 \mathrm{e}-09$ & $2.1 \mathrm{e}-10$ & $3.7 e-11$ & $1.4 \mathrm{e}-12$ & $8.5 e-13$ & $1.9 \mathrm{e}-14$ & $4.1 \mathrm{e}-16$ & $1.3 e-16$ \\
\hline 3 & $1.6 \mathrm{e}-05$ & $9.5 e-11$ & $1.0 \mathrm{e}-09$ & $8.5 e-10$ & 7.0e-12 & $1.2 \mathrm{e}-11$ & $5.0 \mathrm{e}-13$ & $6.5 e-14$ & $2.3 e-16$ & $1.2 \mathrm{e}-16$ & $1.6 \mathrm{e}-17$ & $7.1 \mathrm{e}-22$ \\
\hline 4 & $1.6 \mathrm{e}-05$ & $1.2 \mathrm{e}-07$ & $1.9 \mathrm{e}-09$ & $5.2 \mathrm{e}-11$ & $2.2 \mathrm{e}-12$ & $8.9 \mathrm{e}-13$ & $2.0 \mathrm{e}-14$ & $2.8 \mathrm{e}-16$ & $1.0 \mathrm{e}-17$ & $1.9 \mathrm{e}-19$ & $1.2 \mathrm{e}-20$ & $8.8 \mathrm{e}-23$ \\
\hline 5 & $4.9 \mathrm{e}-06$ & $7.0 \mathrm{e}-07$ & $2.1 \mathrm{e}-08$ & $5.0 \mathrm{e}-12$ & $6.9 \mathrm{e}-14$ & $2.2 \mathrm{e}-14$ & $3.3 e-17$ & $1.7 \mathrm{e}-17$ & $2.0 \mathrm{e}-19$ & $6.0 \mathrm{e}-21$ & $4.0 \mathrm{e}-24$ & $8.2 \mathrm{e}-25$ \\
\hline 6 & $2.6 \mathrm{e}-06$ & $1.0 \mathrm{e}-06$ & $2.8 \mathrm{e}-08$ & $4.5 \mathrm{e}-11$ & $4.5 e-13$ & $3.5 e-15$ & $3.6 \mathrm{e}-18$ & $1.9 \mathrm{e}-18$ & $9.3 e-21$ & $4.5 e-23$ & $3.3 e-25$ & $2.0 \mathrm{e}-27$ \\
\hline 7 & $4.7 \mathrm{e}-06$ & $8.8 \mathrm{e}-07$ & $8.5 \mathrm{e}-09$ & $4.5 \mathrm{e}-10$ & $2.7 \mathrm{e}-12$ & $7.7 \mathrm{e}-16$ & $1.6 \mathrm{e}-18$ & $6.9 \mathrm{e}-20$ & $2.1 \mathrm{e}-23$ & $2.5 e-24$ & $8.6 e-27$ & $5.9 \mathrm{e}-29$ \\
\hline
\end{tabular}

Table 2: Estimated extrapolation error, using formula (20), in the approximation of the rotation number of the shearless curve of the map (34) that corresponds to $a=0.615$ and $b=0.4$.
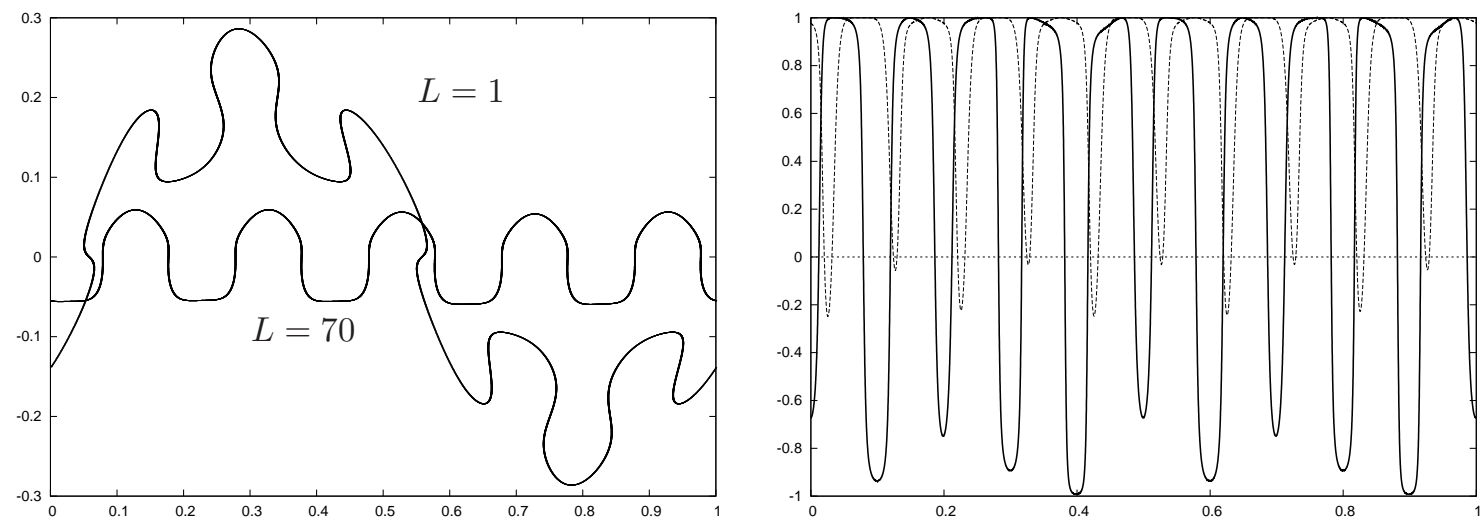

Figure 9: Left: we plot in the $x y$-plane the shearless curve $\gamma_{S}=\gamma^{\left(1, \theta_{0}\right)}$ in Figure 8 and the averaged curve $\gamma^{\left(70, \theta_{0}\right)}$, where $\theta_{0}=\Theta_{21,7}$. Right: we plot function (30) on the previous curves versus the arc parameter $\alpha$ on $\mathbb{T}$ described in Remark 3.1.

Remark 3.2. Of course, the points in the left plot of Figure 8 to which we assign a rational rotation number (the profile is locally constant) cannot belong to an invariant curve. These points correspond to "secondary invariant curves" or "islands", which are invariant curves of a suitable power of the map, that appear close to the elliptic periodic orbits. Thus, for a point on these islands, what we obtain is the "winding number" of the periodic orbit in the middle of the island. We refer to discussions in [23, 29].

Now, let us illustrate the methodology of Section 2 in order to unfold the shearless invariant curve $\gamma_{S}$. To this end, we complexify phase space by means of the change of variables $z=$ $e^{y} e^{2 \pi \mathrm{i} x}$ and compute the new quasi-periodic signal $\left\{z_{n}^{\left(L, \theta_{0}\right)}\right\}$ using the approximation $\theta_{0}=\Theta_{21,7}$. In Figure 9 (left plot) we show the original curve $\gamma_{S}$, corresponding to $L=1$, together with the curve $\gamma^{\left(70, \theta_{0}\right)}$ that is less folded but its projection is not well defined yet. In Figure 9 (right plot) we show function (30) for $\gamma_{S}$ and $\gamma^{\left(70, \theta_{0}\right)}$. Actually, the projection into a circle is well posed for $L=75$ and the curve is very close to be a circle - the minimum of function (30) is $\simeq 0.999897$ - for $L=240$.

Furthermore, we try to unfold $\gamma_{S}$ using higher order averages to accelerate the convergence to a circle. To this end, we still fix $\theta_{0}=\Theta_{21,7}$ and apply the higher order method explained in 


\begin{tabular}{|cccccc|}
\hline$q$ & $p=1$ & $p=2$ & $p=3$ & $p=4$ & $p=5$ \\
\hline 6 & $2.8 \mathrm{e}-02(-)$ & $5.6 \mathrm{e}-02(-)$ & $7.1 \mathrm{e}-02(-)$ & $7.5 \mathrm{e}-02(-)$ & $7.4 \mathrm{e}-02(-)$ \\
7 & $1.1 \mathrm{e}-01(+)$ & $1.5 \mathrm{e}-01(=)$ & $1.2 \mathrm{e}-01(=)$ & $7.7 \mathrm{e}-02(-)$ & $4.2 \mathrm{e}-02(-)$ \\
8 & $4.0 \mathrm{e}-02(+)$ & $5.6 \mathrm{e}-02(+)$ & $9.6 \mathrm{e}-02(=)$ & $1.4 \mathrm{e}-01(=)$ & $1.4 \mathrm{e}-01(=)$ \\
9 & $1.8 \mathrm{e}-03(+)$ & $4.4 \mathrm{e}-03(+)$ & $3.4 \mathrm{e}-02(+)$ & $6.7 \mathrm{e}-02(+)$ & $6.4 \mathrm{e}-02(=)$ \\
10 & $2.8 \mathrm{e}-03(+)$ & $3.6 \mathrm{e}-05(+)$ & $7.8 \mathrm{e}-03(+)$ & $1.2 \mathrm{e}-02(+)$ & $1.1 \mathrm{e}-02(+)$ \\
11 & $2.0 \mathrm{e}-03(+)$ & $3.5 \mathrm{e}-05(+)$ & $1.0 \mathrm{e}-06(+)$ & $2.6 \mathrm{e}-04(+)$ & $1.9 \mathrm{e}-03(+)$ \\
12 & $3.0 \mathrm{e}-03(+)$ & $2.1 \mathrm{e}-04(+)$ & $7.6 \mathrm{e}-06(+)$ & $2.4 \mathrm{e}-06(+)$ & $1.3 \mathrm{e}-04(+)$ \\
13 & $9.6 \mathrm{e}-04(+)$ & $1.1 \mathrm{e}-04(+)$ & $3.0 \mathrm{e}-06(+)$ & $9.8 \mathrm{e}-08(+)$ & $1.5 \mathrm{e}-08(+)$ \\
14 & $8.0 \mathrm{e}-04(+)$ & $2.0 \mathrm{e}-05(+)$ & $3.8 \mathrm{e}-07(+)$ & $7.7 \mathrm{e}-08(+)$ & $4.5 \mathrm{e}-09(+)$ \\
15 & $3.0 \mathrm{e}-04(+)$ & $3.5 \mathrm{e}-06(+)$ & $1.7 \mathrm{e}-07(+)$ & $1.9 \mathrm{e}-08(+)$ & $8.0 \mathrm{e}-10(+)$ \\
16 & $2.0 \mathrm{e}-05(+)$ & $8.4 \mathrm{e}-07(+)$ & $1.9 \mathrm{e}-08(+)$ & $9.3 \mathrm{e}-10(+)$ & $1.8 \mathrm{e}-11(+)$ \\
\hline \hline
\end{tabular}

Table 3: Estimated distance to be a circle of the higher order averaged curve $\gamma^{\left(2^{q}, \theta_{0}, p\right)}$, where $\theta_{0}=\Theta_{21,7}$, for the shearless curve $\gamma_{S}$ in Figure 8. The meaning of the symbols is the following: (-) if the curve is still folded, $(=)$ if the curve is unfolded but not close enough to a circle and $(+)$ if the curve is close to be a circle.
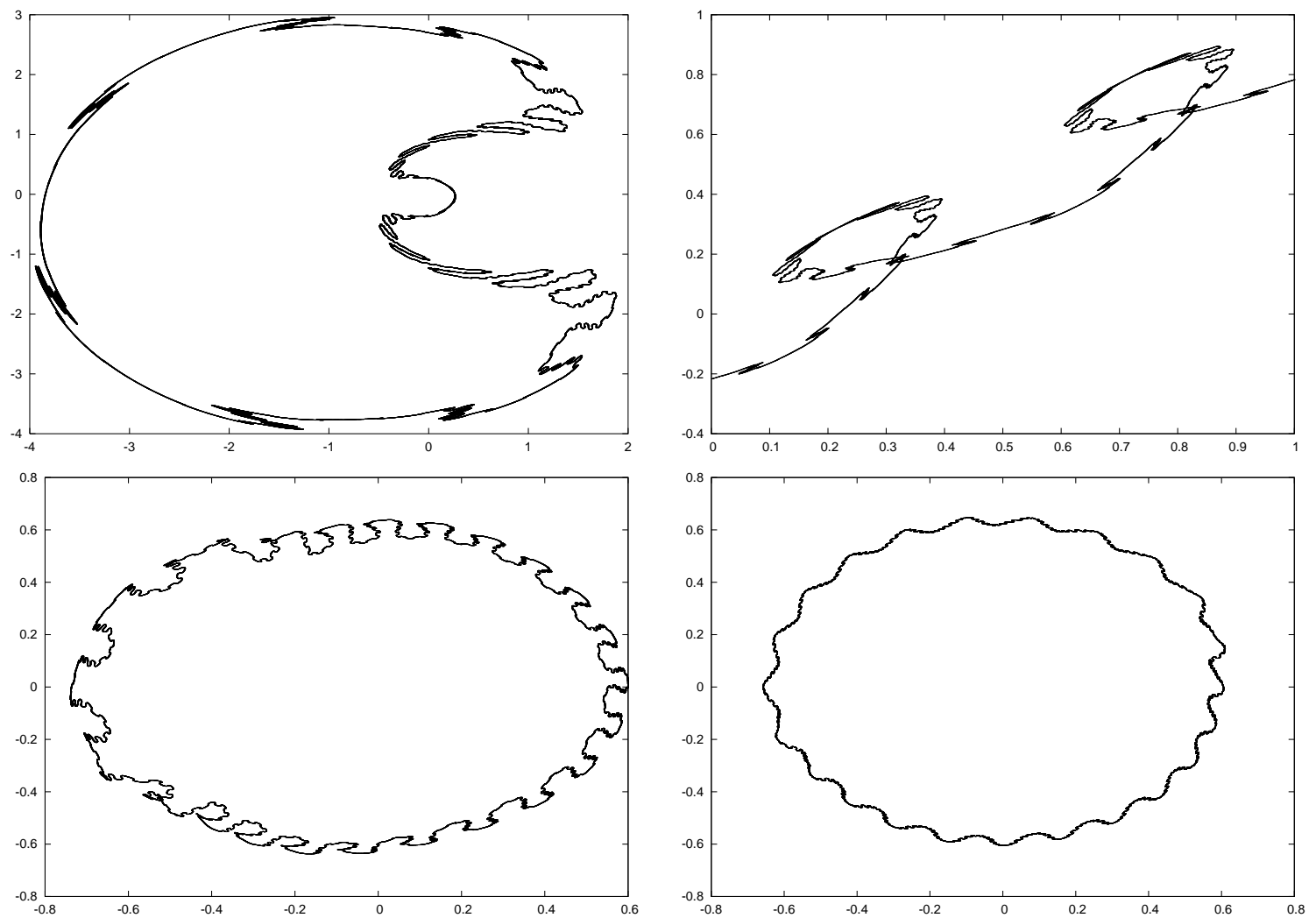

Figure 10: Top-left: Higher order meandering curve for the standard nontwist map in the complexified phase space using $z=\mathrm{e}^{\sqrt{2 \pi|a|} y} \mathrm{e}^{2 \pi \mathrm{i} x}$. Top-right: Circle correspondence obtained from the direct projection - defined in (1) — of the iterates of the curve in top-left plot. Bottom: We show the curves $\gamma^{\left(L, \theta_{0}\right)}$, where $\theta_{0}=0.0429853252$, for $L=50$ (left) and $L=150$ (right) in the complex plane.

Section 2.6 to unfold the curve, using different values of the extrapolation order $p$ and number 

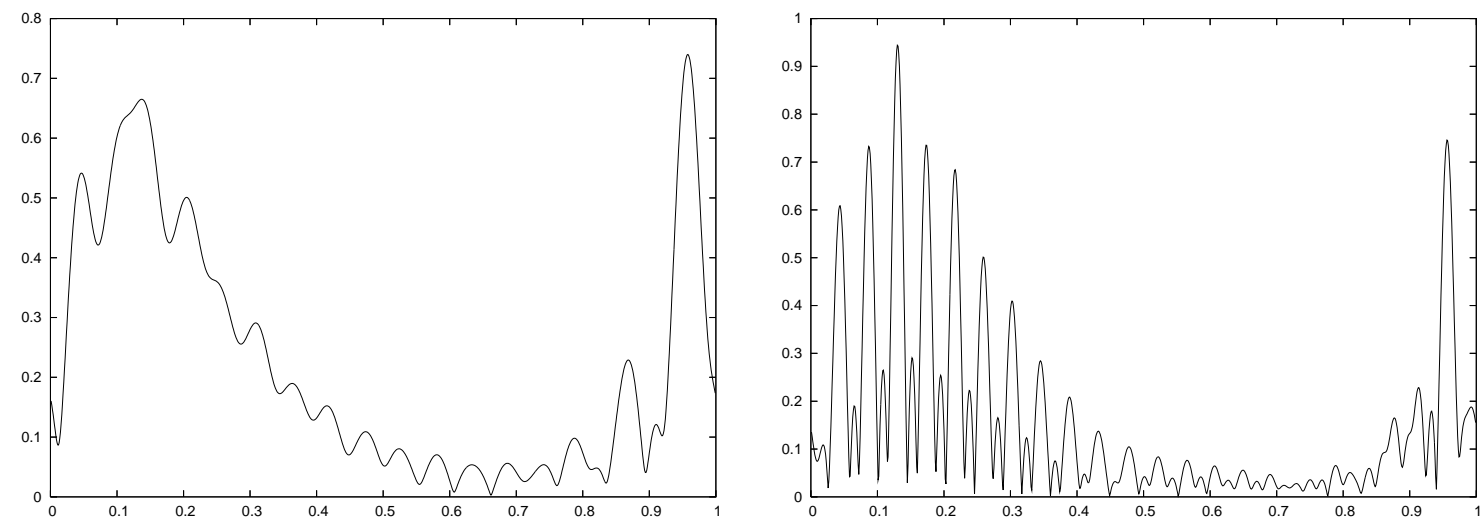

Figure 11: We plot the function $\theta_{0} \mapsto\left|z_{0}^{\left(L, \theta_{0}\right)}\right|$ using the values $L=30$ (left) and 60 (right) for the meandering curve in Figure 10. The real value of the rotation number is around 0.04332244074906551 (see the text for details).

of iterates $L=2^{q}$. In table 3 we present the estimated error when we compare the new curve with a circle. Since we have constructed a sequence of curves tending to a circle —up to small error- we estimate the distance of these curves to be a circle just looking at the number of digits that coincide for a point on these curves when we increase the number of iterates from $2^{q-1}$ to $2^{q}$, this is, we use the formula $\left|z_{0}^{\left(2^{q}, \theta_{0}, p\right)}-z_{0}^{\left(2^{q-1}, \theta_{0}, p\right)}\right|$, where $\theta_{0}=\Theta_{21,7}$. The adjacent symbol in this table indicates if the projection of the invariant curve induces a circle map, according to the criterion of the sign of function (30) - as explained in Remark 3.1. We put the symbol (-) if the minimum of function (30) is negative, we put $(=)$ if this minimum is positive but less than 0.99 and we put $(+)$ if this minimum is between 0.99 and 1 . As expected, for $p=1$ and $q=6$ the curve is still folded since $z_{n}^{\left(2^{q}, \theta_{0}\right)}=z_{n}^{\left(2^{q}, \theta_{0}, 1\right)}$ and $2^{6}=64<75$. Let us observe that when we increase the order of averaging we require more iterates in order to appreciate an improvement in the extrapolation. Nevertheless, after this transition the method turns out to be much more efficient (for example, see the row for $q=16$ ).

A further step is to consider the case of the so called higher order meanderings that appear due to reconnections involving periodic orbits in a neighborhood of a meandering curve. The considered example is selected from [34] and we refer there for a constructive explanation. Concretely, we consider the values $a=-0.071963192$ and $b=-0.44614508325727$ and the curve that corresponds to the initial condition $\left(x_{0}, y_{0}\right)=(0,-2.00377736103447)$. In Figure 10 (left plot) we show this higher order meandering curve in the complex plane by means of the change of variables $z=\mathrm{e}^{\sqrt{2 \pi|a|} y} \mathrm{e}^{2 \pi \mathrm{i} x}$.

Let us observe that the invariant curve of this example is folded in a very wild way. Actually, in the top-right plot of Figure 10 we show the "lift" to $\mathbb{R}$ of the circle correspondence that we obtain by means of the direct projection on the angular variable $x$. This plot has been obtained projecting iterates of an orbit of the curve, and we observe that it is not easy to compute numerically this lift just looking at isolated iterates of the circle correspondence - without any "a-priori" information on the rotation number. Therefore, we apply Laskar's method of 


\begin{tabular}{|ll|ll|ll|ll|ll|}
\hline \multicolumn{2}{|c|}{$L=30$} & \multicolumn{3}{c|}{$L=60$} & \multicolumn{2}{c|}{$L=90$} & \multicolumn{2}{c|}{$L=120$} & \multicolumn{3}{c|}{$L=150$} \\
\hline 0.9577778096 & $(0.74)$ & 0.1303407362 & $(0.95)$ & 0.1302228135 & $(0.86)$ & 0.1302697604 & $(0.82)$ & 0.1301364744 & $(0.84)$ \\
0.1371713373 & $(0.67)$ & 0.9562939918 & $(0.75)$ & 0.9565703718 & $(0.77)$ & 0.9565427675 & $(0.72)$ & 0.9569018161 & $(0.70)$ \\
$\mathbf{0 . 0 4 6 5 9 5 9 0 5 6}$ & $(0.54)$ & 0.1734756047 & $(0.74)$ & 0.0870283875 & $(0.68)$ & 0.0870160838 & $(0.62)$ & 0.0865975344 & $(0.65)$ \\
0.2046548291 & $(0.50)$ & 0.0872525690 & $(0.73)$ & 0.1733546296 & $(0.64)$ & $\mathbf{0 . 0 4 3 7 6 9 7 4 1 5}$ & $(0.59)$ & $\mathbf{0 . 0 4 2 9 8 5 3 2 5 2}$ & $(0.62)$ \\
0.3084897181 & $(0.29)$ & 0.2166379341 & $(0.68)$ & $\mathbf{0 . 0 4 3 7 2 3 1 3 5 2}$ & $(0.61)$ & 0.1737224972 & $(0.58)$ & 0.1736243983 & $(0.61)$ \\
0.8683260095 & $(0.23)$ & $\mathbf{0 . 0 4 3 5 6 7 9 4 2 2}$ & $(0.61)$ & 0.2169984090 & $(0.58)$ & 0.2172096615 & $(0.54)$ & 0.2171559900 & $(0.57)$ \\
0.3631744037 & $(0.19)$ & 0.2593740449 & $(0.50)$ & 0.2601564576 & $(0.42)$ & 0.2604187823 & $(0.38)$ & 0.2606176626 & $(0.40)$ \\
0.4154453760 & $(0.15)$ & 0.3024868348 & $(0.41)$ & 0.3037390964 & $(0.34)$ & 0.3041163591 & $(0.32)$ & 0.3041431703 & $(0.34)$ \\
\hline
\end{tabular}

Table 4: Relevant maxima of the function $\theta_{0} \in[0,1] \mapsto\left|z_{0}^{\left(L, \theta_{0}\right)}\right|$ for different values of $L$ corresponding to the invariant curve in Figure 10 (top-left plot). In parentheses we show the value of the function at the local maxima. We write in bold the value of the maximum that approximates the rotation number of the curve.

\begin{tabular}{|c|cccccccc|c|}
\hline$i$ & $k_{i 1}$ & $k_{i 2}$ & $k_{i 3}$ & $k_{i 4}$ & $k_{i 5}$ & $k_{i 6}$ & $k_{i 7}$ & $k_{i 8}$ & $\kappa_{i}$ \\
\hline 1 & 1 & -8 & 9 & -7 & -6 & 8 & 2 & 10 & 51 \\
2 & -3 & 1 & -4 & -2 & -5 & -1 & -6 & -7 & 29 \\
3 & -5 & -6 & 1 & -11 & 7 & 6 & 13 & -4 & 53 \\
4 & -10 & -12 & 2 & 1 & -9 & -11 & 3 & -8 & 56 \\
5 & -4 & 9 & 10 & 5 & 1 & -9 & -8 & 6 & 52 \\
6 & 3 & -1 & 4 & 2 & 5 & 1 & 6 & 7 & 29 \\
7 & -11 & -4 & -7 & 8 & -3 & 4 & 1 & 5 & 43 \\
8 & 7 & -10 & -6 & -3 & 4 & 10 & -9 & 1 & 50 \\
\hline
\end{tabular}

Table 5: Indicators $k_{i j}$ and $\kappa_{i}$ corresponding to the step "rotation number selection" described in Section 2.4. We use $L=60$.

frequency analysis (implemented as described in Section 2.4) to obtain a sufficiently good approximation of the rotation number in order to unfold the curve. Maxima chasing is performed looking for local maxima of $\theta_{0} \mapsto\left|z_{0}^{\left(L, \theta_{0}\right)}\right|$ in the interval $[0,1]$. These maxima are obtained using a partition of 500 points of this interval and asking for a tolerance $\varepsilon_{G S S}=10^{-10}$ in the golden section search. In Table 4 we show the revelant maxima, using $\nu=5$ in equation (13), for several values of $L$. Moreover, in Figure 11 we plot the function $\theta_{0} \mapsto\left|z_{0}^{\left(L, \theta_{0}\right)}\right|$, for $L=30$ (left plot) and $L=60$ (right plot). Of course, when we increase $L$ we observe a larger number of maxima but the width of the peaks is reduced so their approximation is improved.

As explained in Section 2.4, we assume that one of these maxima approximates the rotation number of the curve in such a way that the rest are multiples of it (modulo 1). To select which maximum approximates the rotation number, we compute the indicators $k_{i j}, \kappa_{i}, d_{i j}$ and $\delta_{i}$ given in equations (14) and (15). For example, the values corresponding to $L=60$ are given in Tables 5 and 6 . Let us point out that the maximum corresponding to the $i$-th row in these tables can be read in the $i$-th row of Table 4. If we select the 2 nd or the 6 th maxima as the approximation $\theta_{0}$ of the rotation number, then the remaining peaks are approximated — with the error shown in Table 6- as multiples $k \theta_{0}$ (modulo 1) using smaller values for $k$ than if we make any other choice (see Table 5). These two peaks correspond to approximations of $1-\theta$ and $\theta$, and we select the 6 th one as an approximation of the rotation number since is follows the positive orientation of the dynamics. We write this peak in bold in Table 4.

Let us refine the approximation of the rotation number from the 6 th peak. In Figure 10 


\begin{tabular}{|c|cccccccc|c|}
\hline$i$ & $d_{i 1}$ & $d_{i 2}$ & $d_{i 3}$ & $d_{i 4}$ & $d_{i 5}$ & $d_{i 6}$ & $d_{i 7}$ & $d_{i 8}$ & $\delta_{i}$ \\
\hline 1 & $0.00 \mathrm{e}+00$ & $9.80 \mathrm{e}-04$ & $4.00 \mathrm{e}-04$ & $3.60 \mathrm{e}-04$ & $1.30 \mathrm{e}-03$ & $8.40 \mathrm{e}-04$ & $1.30 \mathrm{e}-03$ & $9.20 \mathrm{e}-04$ & $6.10 \mathrm{e}-03$ \\
2 & $7.70 \mathrm{e}-04$ & $0.00 \mathrm{e}+00$ & $1.30 \mathrm{e}-03$ & $1.50 \mathrm{e}-04$ & $1.80 \mathrm{e}-03$ & $1.30 \mathrm{e}-04$ & $2.80 \mathrm{e}-03$ & $3.40 \mathrm{e}-03$ & $1.00 \mathrm{e}-02$ \\
3 & $2.20 \mathrm{e}-03$ & $2.80 \mathrm{e}-03$ & $0.00 \mathrm{e}+00$ & $4.50 \mathrm{e}-03$ & $2.30 \mathrm{e}-03$ & $2.70 \mathrm{e}-03$ & $4.10 \mathrm{e}-03$ & $3.60 \mathrm{e}-03$ & $2.20 \mathrm{e}-02$ \\
4 & $2.80 \mathrm{e}-03$ & $3.30 \mathrm{e}-03$ & $1.00 \mathrm{e}-03$ & $0.00 \mathrm{e}+00$ & $1.90 \mathrm{e}-03$ & $3.30 \mathrm{e}-03$ & $2.30 \mathrm{e}-03$ & $5.00 \mathrm{e}-04$ & $1.50 \mathrm{e}-02$ \\
5 & $3.10 \mathrm{e}-03$ & $6.50 \mathrm{e}-03$ & $7.10 \mathrm{e}-03$ & $4.00 \mathrm{e}-03$ & $0.00 \mathrm{e}+00$ & $6.60 \mathrm{e}-03$ & $7.50 \mathrm{e}-03$ & $2.60 \mathrm{e}-03$ & $3.70 \mathrm{e}-02$ \\
6 & $3.60 \mathrm{e}-04$ & $1.30 \mathrm{e}-04$ & $7.90 \mathrm{e}-04$ & $1.10 \mathrm{e}-04$ & $1.20 \mathrm{e}-03$ & $0.00 \mathrm{e}+00$ & $2.00 \mathrm{e}-03$ & $2.40 \mathrm{e}-03$ & $7.10 \mathrm{e}-03$ \\
7 & $1.60 \mathrm{e}-02$ & $6.20 \mathrm{e}-03$ & $1.00 \mathrm{e}-02$ & $1.20 \mathrm{e}-02$ & $5.20 \mathrm{e}-03$ & $6.00 \mathrm{e}-03$ & $0.00 \mathrm{e}+00$ & $5.60 \mathrm{e}-03$ & $6.20 \mathrm{e}-02$ \\
8 & $1.20 \mathrm{e}-02$ & $1.80 \mathrm{e}-02$ & $1.10 \mathrm{e}-02$ & $5.20 \mathrm{e}-03$ & $6.60 \mathrm{e}-03$ & $1.80 \mathrm{e}-02$ & $1.80 \mathrm{e}-02$ & $0.00 \mathrm{e}+00$ & $9.20 \mathrm{e}-02$ \\
\hline
\end{tabular}

Table 6: Indicators $d_{i j}$ and $\delta_{i}$ corresponding to the step "rotation number selection" described in Section 2.4. We use $L=60$.

we plot $\gamma^{\left(L, \theta_{0}\right)}$ using the approximation $\theta_{0}=0.0429853252$ for $L=50$ (bottom-left plot) and $L=150$ (bottom-right plot). We observe that for $L=50$ the curve is still very folded but if we take $L=150$, even thought there are still some harmonics that fold the curve, we are close to a circle centered at the origin and the projection gives us a circle correspondence that we can "lift" to $\mathbb{R}$ easily, since the size of the folds is small when compared with $\theta_{0}$. Finally, we compute the rotation number of this invariant curve from the circle correspondence obtained by means of this unfolding procedure. We apply Algorithm 2.14, using $p=7$ and $q=21$, to the iterates $x_{n}=\arg \left(z_{n}^{\left(150, \theta_{0}\right)}\right) / 2 \pi$ thus obtaining the approximation $\Theta_{7,21}=0.04332244074906551$ with an estimated error $1.7 \cdot 10^{-13}$.

We observe that the computation of the rotation number does not work as well as in previous examples, but an error of order $10^{-13}$ is very satisfying in this context since a huge number of Fourier coefficients is required to approximate the curve with this error and the rotation number is close to resonance (see also the discussion regarding Hénon's map example). To show this, let us compute the Fourier coefficients corresponding to $|k| \leq 750$ by means of Algorithm 2.20 using $p=7, q=21$ and $\theta \simeq \Theta_{21,7}$. Computations are performed using 32-digit arithmetics (double-double data type from [18]). The modulus of the obtained values $\Phi_{21,7, k}$ are shown in the left plot of Figure 12. The extrapolation error, estimated using (29), typically ranges between $10^{-10}$ and $10^{-8}$. As expected, the decay of these coefficients is very mild and we point out that coefficients for $|k| \simeq 750$ are still of order $10^{-5}$. It is interesting to compare this behavior to that corresponding to the shearless curve in Figure 8 since the extrapolation methods can be applied successfully to this case. For this curve the Fourier coefficients - computed using the same implementation parameters - decay much faster (see the right plot in Figure 12). In this case, the estimated extrapolation error typically ranges between $10^{-16}$ and $10^{-24}$, and Fourier coefficients for $|k| \geq 400$ are so small that we cannot compute any significant digit (we detect this fact because $\left|\Phi_{21,7, k}\right|$ is of the same order as the extrapolation error). A final remark is that in this plot we observe an increase of the size of Fourier coefficients around $|k| \simeq 250$ and after that they decay again at the same rate. The rotation number of this curve has the convergent $145 / 242$, so the small divisor for the corresponding Fourier coefficient turns out to be very small. Precisely we observe that $\left|\Phi_{21,7,243}\right| \simeq 214\left|\Phi_{21,7,242}\right|$.

In the next example we want to illustrate the computation of derivatives of the rotation number by applying Algorithm 2.16 to a circle correspondence that we can "lift" numerically to 

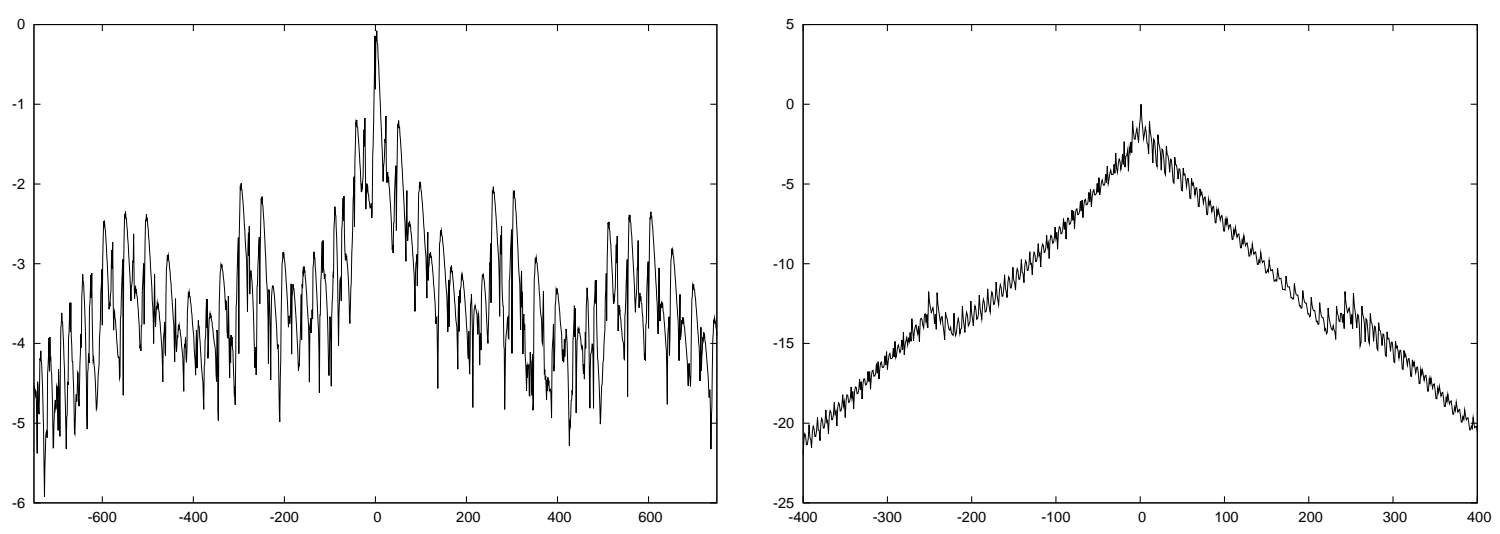

Figure 12: We plot $\log _{10}\left|\Phi_{21,7, k}\right|$ versus $k$ corresponding to the approximated Fourier coefficients of two studied invariant curves. Left: Higher order meandering curve in Figure 10 (top-left plot). Right: Shearless invariant curve in Figure 8 (left plot).

$\mathbb{R}$. Moreover, we also want to stress how extrapolation methods are less accurate when the rotation number is a Diophantine number "close to a rational" - thus having a large constant $C$ in (7). Therefore, we select a problem very close to a resonance. Comparing with previous examples, we point out that the shearless curve in Figure 8 is not too resonant - actually the continued fraction of the rotation number in that case is $[0,1,1,2,48,1, \ldots]$. Meanwhile the case of higher order meandering in Figure 10 is more resonant but the curve extremely complicated. Let us consider the well-known Hénon family, which is a paradigmatic example since it appears generically in the study of a saddle-node bifurcation. This family can be written as

$$
H_{\alpha}:\left(\begin{array}{l}
u \\
v
\end{array}\right) \longmapsto\left(\begin{array}{cc}
\cos (2 \pi \alpha) & -\sin (2 \pi \alpha) \\
\sin (2 \pi \alpha) & \cos (2 \pi \alpha)
\end{array}\right)\left(\begin{array}{c}
u \\
v-u^{2}
\end{array}\right) .
$$

It is not difficult to check that we can perform a close to the identity change of variables to guarantee the twist condition close to the origin, except for the values $\alpha=1 / 3,2 / 3$. Then, for values of $\alpha$ close to $1 / 3$ and $2 / 3$, reconnection takes places and meandering phenomena arises, i.e., there are folded invariant curves. Next we want to illustrate the computation of the derivatives of the rotation number for $\alpha$ close to $1 / 3$.

In the left plot of Figure 13 we show meandering curves for $\alpha=0.299544$. We use the direct projection and we apply Algorithm 2.16 to the "lift" of the circle correspondence thus obtained, in order to compute the derivative of the rotation number with respect to the initial condition $u_{0}$, for 6000 points of the form $\left(u_{0}, 0.15\right)$ along the dotted line in the figure. Computations are performed taking $p=8, q=23$, and using 32-digit arithmetics (double-double data type from [18]). The corresponding profile is shown in the right plot of Figure 13, and we observe that the sign of the derivative changes when we pass from one twist zone to another. The isolated points where the derivative vanishes correspond to the shearless invariant curve while the points where it is locally constant corresponds to the chain of islands (secondary tori) around 

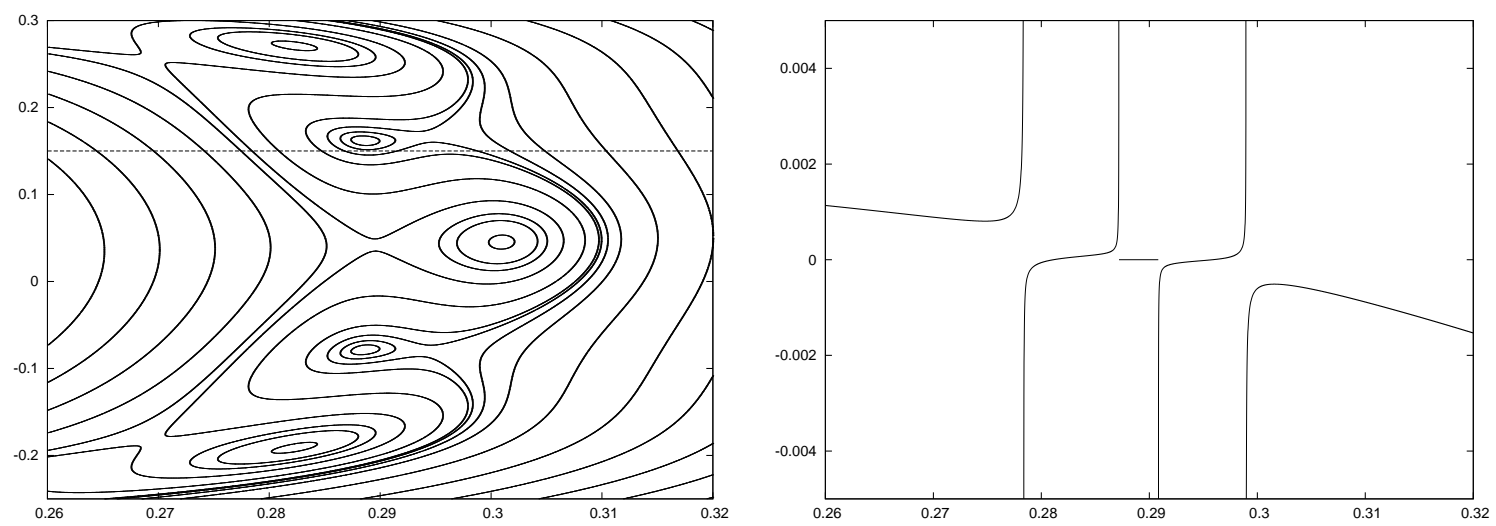

Figure 13: Left: Phase space in the $u v$-plane of Hénon map for $\alpha=0.299544$ showing meandering curves close to periodic orbits of period 3/10. Right: we plot the derivative of the rotation number $u \mapsto D_{u} \rho$ along the straight line in the left plot.

the elliptic periodic points (see Remark 3.2). We want to stress that since we are very close to a resonance, the convergence of the computations in this example is not as good as in the far-from-resonance case. For example, for $u_{0}=0.28$ we obtain the approximation $\Theta_{23,8}=$ 0.299999020519 of the rotation number with an estimated error, using (20), of order $10^{-12}$ and the derivative $\Theta_{23,8,7}^{1}=-6.027735852 \cdot 10^{-5}$ with an estimated error, using (22), of order $10^{-10}$. Actually, the continued fraction expansion of $\Theta_{23,8}$ is given by $[0,3,2,1,10203,2, \ldots]$ which is close to $3 / 10$ (winding number of the periodic orbit). It is interesting to compare the error of order $10^{-12}$ in the computation of the rotation number with the error obtained when dealing with good Diophantine numbers, that are typically of order $10^{-30}$ for the used implementation parameters. We refer to several comments given in $[23,29]$ regarding this situation.

We remark that all the previous examples considered in this section contain only one harmonic or are written as perturbations of maps that have strong twist behavior. For this reason we have not shown all the possibilities of our methodology. As it is pointed out in [34], folded invariant curves appear in a natural way, when we introduce more harmonics in the studied family of maps. Since these curves can be constructed following arbitrarily complicated paths in phase space, they got the name of labyrinthic curves. We shall consider the following family of maps $F_{\varepsilon}=F_{s_{1}, s_{2}, c_{1}, y_{2}, y_{3}, \gamma, \varepsilon}$ defined on $\mathbb{R} /(2 \pi \mathbb{Z}) \times \mathbb{R}$ :

$$
F_{\varepsilon}:\left(\begin{array}{c}
x \\
y
\end{array}\right) \longmapsto\left(\begin{array}{c}
\bar{x} \\
\bar{y}
\end{array}\right)=\left(\begin{array}{c}
x+\varepsilon P(\bar{y}) \\
y+\varepsilon \gamma T(x)
\end{array}\right)
$$

where

$$
\begin{aligned}
& P(y)=y\left(y-y_{2}\right)\left(y-y_{3}\right)(y-1) \\
& T(x)=s_{1} \sin (x)+s_{2} \sin (2 x)+c_{1}(\cos (x)-\cos (2 x)) .
\end{aligned}
$$



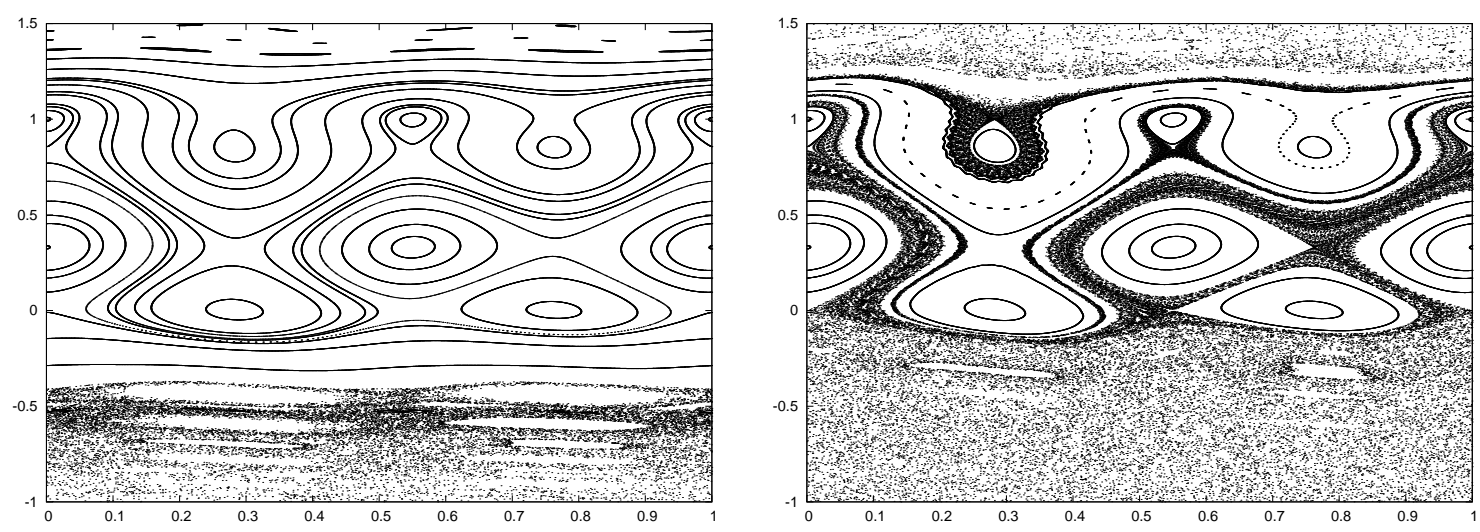

Figure 14: Phase space in the $x y$-plane of family (35). Left: $\varepsilon=5$. Right: $\varepsilon=10$.

From now on, following [34], we fix

$$
\begin{aligned}
y_{2} & =0.33040195 \\
y_{3} & =0.84999789, \\
s_{1} & =0.1608819674465999, \\
s_{2} & =0.9444712344787136, \\
c_{1} & =0.2865154093461046, \\
\gamma & =-0.0049 .
\end{aligned}
$$

In the left plot of Figure 14 we show some iterates for $\varepsilon=5$ corresponding to several initial conditions. We observe that, if we consider the main elliptic islands as holes on the cylinder, then we find invariant curves of different homotopy classes. Since these curves are folded in a very complicated way, it is difficult to face the systematic computation of the "lift" of the direct projection of the iterates. Then, the unfolding method turns out to be very useful to computate these invariant curves.

For example, we consider the curve associated to the initial condition $\left(x_{0}, y_{0}\right)=(0,2.2)$, for $\varepsilon=5$. We first obtain an approximation of the rotation number by means of frequency analysis as it is explained in Section 2.4. In particular, using the complex variable $z=\mathrm{e}^{y} \mathrm{e}^{\mathrm{i} x}$ and $L=100$ we obtain the approximation of the rotation number $\theta_{0}=7.46161 \cdot 10^{-3}$ (details are omitted since they are very similar of those corresponding to the previously discussed examples). Using this approximation $\theta_{0}$ we can unfold the invariant curve computing the averages ( 3 ) for $L=150$ (for this values the curve is almost a circle). Then, we apply Algorithm 2.14 using $p=9$ and $q=23$. Computations are performed using 32-digit arithmetics (double-double data type from [18]). We obtain the approximation

$$
\theta \simeq \Theta_{23,9}=0.0080998168999841202002324221453501
$$

of the rotation number with an estimated extrapolation error of order $10^{-34}$, that corresponds with the arithmetic precision of our computations. Let us observe that the number (36) is very 

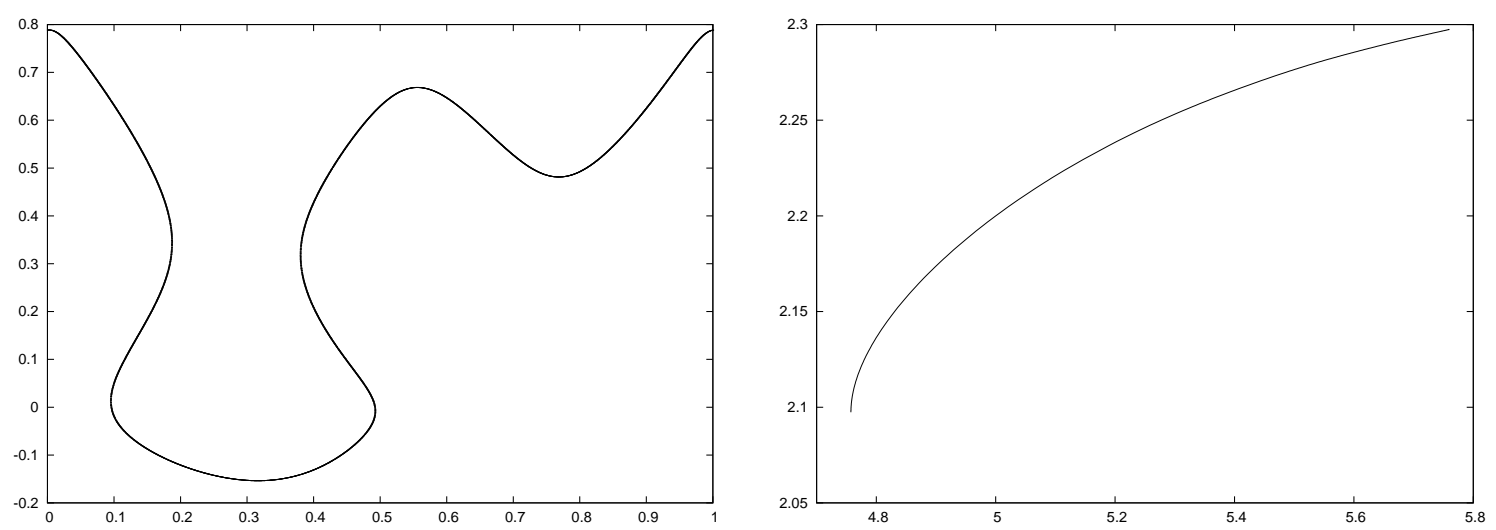

Figure 15: Left: Labyrinthic invariant curve in the $x y$-plane of (35) corresponding to the initial condition $\left(x_{0}, y_{0}\right)=(0,2.2)$ for $\varepsilon=5$. Right: We plot $y(\varepsilon)$ versus $\varepsilon$, in the continuation of the invariant curve on the left.

close to zero, but its continued fraction is $[0,123,2,5,1,2, \ldots]$, so we conclude that it is not so resonant as the example of Hénon's map that we considered before - for this reason the obtained computations are more accurate.

Furthermore, we use the methods of the paper to follow the evolution, when $\varepsilon$ changes, of the invariant curve of $F_{\varepsilon}$ having a prefixed rotation number $\theta$. Concretely, let $\left(x_{0}, y\left(\varepsilon_{0}\right)\right)$ be a point on an invariant curve of rotation number $\theta$ of the map $F_{\varepsilon_{0}}$. Then, given $\varepsilon$ close to $\varepsilon_{0}$, we want to compute an initial condition $\left(x_{0}, y(\varepsilon)\right)$ that corresponds to the invariant curve -if it exists - of $F_{\varepsilon}$ that has the same rotation number. Indeed, if we denote by $\rho\left(\varepsilon, y_{0}\right)$ the function that gives the rotation number of the invariant curve of $F_{\varepsilon}$ for the point $\left(x_{0}, y_{0}\right)$-if it exists - then we look for solutions with respect to $y_{0}=y(\varepsilon)$ of the equation $\rho\left(\varepsilon, y_{0}\right)=\theta$. The computation of the point $y(\varepsilon)$ is performed by means of the secant method as it is done in [29] for the standard map. Another possibility is to use a Newton scheme like in [23] for Hénon's map, computing derivatives of the rotation number as described in Section 2.5, but the secant method is enough for our purposes.

We continue the curve of rotation number given by (36) starting from the values $\varepsilon_{0}=5$ and $\left(x_{0}, y_{0}\right)=(0,2.2)$. Computations are performed using 32-digit arithmetics (double-double data type from [18]). In order to unfold invariant curves we use as an approximation the prefixed value of the rotation number (curves nearby have a similar rotation number) and we take $L=$ 150. For computing the rotation number, we apply Algorithm 2.14 using $p=6$ and $2^{16}$ iterates of the map at most. We estimate the error of the rotation number by using (20) and we validate this computation if it is smaller than $10^{-16}$. For the secant method we require an error smaller than $10^{-14}$.

The continuation of the invariant curve is performed successfully for values of $\varepsilon$ in the interval $(4.75745894,5.75985518)$ —in Figure 15 we plot $y(\varepsilon)$ in this interval. For $\varepsilon=4.75745894$ the invariant curve turns shearless and then it disappears after the collapse with the other invari- 
ant curve of the same rotation number on the other side of the meandering, so we have a turning point in the continuation. For $\varepsilon=5.75985518$ we stop because we are very close to the breakdown of the curve. For $\varepsilon>5.75985518$ we have observed that the chaotic zone that appears at the breakdown of this invariant curve is very narrow and there are still many invariant curves nearby. In the right plot of Figure 14 we include the phase space that corresponds to $\varepsilon=10$ in order to show the chaotic zone that is created when most of the invariant curves around the continued one are destroyed.

\section{Acknowledgements}

We wish to thank Rafael de la Llave and Carles Simó for stimulating discussions and suggestions. We also acknowledge the use of EIXAM, the UPC Applied Math cluster system for research computing (see http: / / www.ma1.upc.edu/eixam/), and in particular Pau Roldán for his support in the use of the cluster. The authors have been partially supported by the Spanish MCyT/FEDER grant MTM2006-00478. Moreover, the research of A. L. has been supported by the Spanish phD grant FPU AP2005-2950 and the Catalan phD grant 2005FI 00155.

\section{References}

[1] A. Apte, R. de la Llave, and N.P. Petrov. Regularity of critical invariant circles of the standard nontwist map. Nonlinearity, 18(3):1173-1187, 2005.

[2] D.K. Arrowsmith and C.M. Place. An introduction to dynamical systems. Cambridge University Press, 1990.

[3] H. Broer and C. Simó. Resonance tongues in Hill's equations: a geometric approach. J. Differential Equations, 166(2):290-327, 2000.

[4] H. Bruin. Numerical determination of the continued fraction expansion of the rotation number. Phys. D, 59(1-3):158-168, 1992.

[5] E. Castellà and À. Jorba. On the vertical families of two-dimensional tori near the triangular points of the bicircular problem. Celestial Mech. Dynam. Astronom., 76(1):35-54, 2000 .

[6] R. de la Llave, A. González, À. Jorba, and J. Villanueva. KAM theory without action-angle variables. Nonlinearity, 18(2):855-895, 2005.

[7] R. de la Llave, G. Huguet, and Y. Sire. Fast numerical algorithms for the computation of invariant tori in Hamiltonian Systems. Preprint available electronically at http: / / www . ma.utexas.edu/mp_arc-bin/mpa?yn=09-2, 2008. 
[8] R. de la Llave and N.P. Petrov. Regularity of conjugacies between critical circle maps: an experimental study. Experiment. Math., 11(2):219-241, 2002.

[9] Rafael de la Llave and Arturo Olvera. The obstruction criterion for non-existence of invariant circles and renormalization. Nonlinearity, 19(8):1907-1937, 2006.

[10] D. del Castillo-Negrete, J.M. Greene, and P.J. Morrison. Area preserving nontwist maps: periodic orbits and transition to chaos. Phys. D, 91(1-2):1-23, 1996.

[11] A. Delshams and R. de la Llave. KAM theory and a partial justification of Greene's criterion for nontwist maps. SIAM J. Math. Anal., 31(6):1235-1269 (electronic), 2000.

[12] C. Golé. Symplectic Twist Maps: Global Variational Techniques. World Scientific Publishing, 2001.

[13] G. Gómez, À. Jorba, C. Simó, and J. Masdemont. Dynamics and mission design near libration points. Vol. III, volume 4 of World Scientific Monograph Series in Mathematics. World Scientific Publishing Co. Inc., River Edge, NJ, 2001. Advanced methods for triangular points.

[14] G. Gómez, À. Jorba, C. Simó, and J. Masdemont. Dynamics and mission design near libration points. Vol. IV, volume 5 of World Scientific Monograph Series in Mathematics. World Scientific Publishing Co. Inc., River Edge, NJ, 2001. Advanced methods for triangular points.

[15] G. Gómez, J.M. Mondelo, and C. Simó. Refined Fourier analysis: procedures, error estimates and applications. Preprint available at http://www. maia.ub.es/dsg/ 2001 / index.html, 2001.

[16] J.M. Greene. A method for determining a stochastic transition. J. Math. Phys., 20:1183$1201,1979$.

[17] M.R. Herman. Sur la conjugaison différentiable des difféomorphismes du cercle à des rotations. Inst. Hautes Études Sci. Publ. Math., (49):5-233, 1979.

[18] Y. Hida, X. Li, and D. H. Bailey. QD (quad-double/double-double computation package), 2005. Available electronically at http://crd.lbl.gov/ dhbailey/mpdist/.

[19] À. Jorba and E. Olmedo. On the computation of reducible invariant tori in a parallel computer. Preprint available electronically at http://www. maia.ub.es/dsg/2008/ index.shtml, 2008.

[20] À. Jorba, R. Ramírez-Ros, and J. Villanueva. Effective reducibility of quasi-periodic linear equations close to constant coefficients. SIAM J. Math. Anal., 28(1):178-188, 1997. 
[21] À. Jorba and C. Simó. On quasi-periodic perturbations of elliptic equilibrium points. SIAM J. Math. Anal., 27(6):1704-1737, 1996.

[22] J. Laskar, C. Froeschlé, and A. Celletti. The measure of chaos by the numerical analysis of the fundamental frequencies. Application to the standard mapping. Phys. D, 56(2-3):253269, 1992.

[23] A. Luque and J. Villanueva. Computation of derivatives of the rotation number for parametric families of circle diffeomorphisms. Phys. D, 237(20):2599-2615, 2008.

[24] L. Michelotti. Intermediate classical dynamics with applications to beam physics. Wiley Series in Beam Physics and Accelerator Technology. John Wiley \& Sons Inc., New York, 1995. A Wiley-Interscience Publication.

[25] A. Olvera and N.P. Petrov. Regularity properties of critical invariant circles of twist maps, and their universality. Preprint 2008, available electronically at http: / / arxiv.org/ abs/nlin. CD/0609024.

[26] Arturo Olvera and Carles Simó. An obstruction method for the destruction of invariant curves. Phys. D, 26(1-3):181-192, 1987.

[27] R. Paskauskas, C. Chandre, and T. Uzer. Dynamical bottlenecks to intramolecular energy flow. Physical Review Letters, 100(8), 2008.

[28] W.H. Press, S.A. Teukolsky, W.T. Vetterling, and B.P. Flannery. Numerical Recipes in C: The Art of Scientific Computing. Cambridge University Press, second edition edition, 2002.

[29] T.M. Seara and J. Villanueva. On the numerical computation of Diophantine rotation numbers of analytic circle maps. Phys. D, 217(2):107-120, 2006.

[30] T.M. Seara and J. Villanueva. Numerical computation of the asymptotic size of the rotation domain for the Arnold family. Phys. D, 238(2):197-208, 2009.

[31] S. Shinohara and Y. Aizawa. Indicators of reconnection processes and transition to global chaos in nontwist maps. Progr. Theoret. Phys., 100(2):219-233, 1998.

[32] C. L. Siegel and J. K. Moser. Lectures on Celestial Mechanics. Springer-Verlag, New York, 1971. Translation by C. I. Kalme, Die Grundlehren der mathematischen Wissenschaften, Band 187.

[33] C. Simó. Effective computations in celestial mechanics and astrodynamics. In Modern methods of analytical mechanics and their applications (Udine, 1997), pages 55-102. Springer, Vienna, 1998. 
[34] C. Simó. Invariant curves of analytic perturbed nontwist area preserving maps. Regul. Chaotic Dyn., 3(3):180-195, 1998. J. Moser at 70.

[35] A.M. Tarquis, J.C. Losada, R.M. Benito, and F. Borondo. Multifractal analysis of tori destruction in a molecular Hamiltonian system. Phys. Rev. E, 65(1):016213, 9, 2002.

[36] M. van Veldhuizen. On the numerical approximation of the rotation number. J. Comput. Appl. Math., 21(2):203-212, 1988.

[37] A. Wurm, A. Apte, K. Fuchss, and P. J. Morrison. Meanders and reconnection-collision sequences in the standard nontwist map. Chaos, 15(2):023108, 13, 2005.

[38] J.C. Yoccoz. Théorème de Siegel, nombres de Bruno et polynômes quadratiques. Astérisque, (231):3-88, 1995. 